1

5 (1) Rosenstiel School of Marine \& Atmospheric Science, University of Miami, Florida

6

7

\author{
A. C. Haza ${ }^{(1, *)}$, T. M. Özgökmen ${ }^{(1)}$, P. Hogan ${ }^{(2)}$
}

September 24, 2016

(2) Naval Research Laboratory, Stennis Space Center, Mississippi

Submitted to Ocean Modelling

Second Revision

\title{
Impact of Submesoscales on Surface Material Distribution in a Gulf of Mexico Mesoscale Eddy
}

$10 \quad$ Corresponding author: Tel.: +1 3054214648, E-mail: ahaza@rsmas.miami.edu 


\begin{abstract}
Understanding material distribution at the ocean's surface is important for a number of applications, in particular for buoyant pollutants such as oil spills. The main tools to estimate surface flows are satellite altimeters, as well as data-assimilative ocean general circulation models (OGCMs). Current-generation altimeter products rely on the geostrophic approximation to derive surface currents. Recent modeling and experimental work revealed existence of ageostrophic submesoscale motions within the upper ocean boundary layer.

The next frontier is how submesoscales influence transport pathways in the upper ocean, which is a multi-scale problem involving the interaction of submesoscale and mesoscale coherent structures. Here we focus on a mesoscale eddy that exhibits submesoscale fluctuations along its rim. The high-resolution OCGM fields are then treated with two filters. A Lanczos filter is applied to velocity fields to remove the kinetic energy over the submesoscales. Then a Gaussian filter is used for the modeled sea surface height to simulate a geostrophic velocity field that would be available from gridded satellite altimeter data. Lagrangian Coherent Structures (LCS) are then generated from full-resolution and filtered fields to compare Lagrangian characteristics corresponding to different realizations of the surface velocity fields.

It is found that while mesoscale currents exert a general control over the pathways of the tracer initially launched in the mesoscale eddy, there is a leak across the mesoscale transport barriers, induced by submesoscale motions. This leak is quantified as $20 \%$ of the tracer when using the submesoscale filter over one month of advection, while it increases to $50 \%$ using the geostrophic velocity field. We conclude that LCS computed from mesoscale surface velocity fields can be considered as a good first-order proxy, but the leakage of material across them in the presence of submesoscales can be significant.
\end{abstract}

Keywords: submesoscale, upper ocean turbulence, multi-scale transport barriers

\title{
1 Introduction
}

Some of the most important scientific problems regarding ocean dynamics are driven by simple questions as such "where do substances come from and where do they go?". These questions are related to problems involving transport of pollutants and biogeochemical tracers in the ocean, as well as search and rescue missions in case of marine or airplane accidents. Tracking biogeochemical tracers moving with the ocean flow field finds use in understanding the global thermohaline circulation (Broecker et al., 1960), which is a slow transport phenomenon occurring at multi-decadal time scales. On the other hand, tracking pollutants may require rapid estimates in order to minimize socio-economic damage, such as in the response efforts to Deepwater Horizon (DwH) oil spill in the Gulf of Mexico (Crone and Tolstoy, 2010) 
or the radioactive leak into the ocean from the Fukushima meltdown (Rypina et al., 2014). Back-tracking debris from airplane accidents over the oceans, such as in the case of MH370 (Normile, 2014), can aid in narrowing down the location of the accident. These types of problems are best approached in the Lagrangian framework, the general methods of which are reviewed in the summary by LaCasce (2008).

Nevertheless, understanding how material moves under the action of ocean currents, or the Lagrangian transport prediction problem, is extremely challenging. There are at least three reasons for this difficulty. The first of these is the recognition that temporal variability of the flow field is very important in how material gets transported within the system. Aref (1984) showed that even spatially-simple flow fields can lead to chaotic mixing under the action of time dependence. Subsequently, a large number of methods have been developed in order to determine skeletons of transport, or so-called Lagrangian Coherent Structures (LCS) that may not be visible from velocity snapshots (Mezić and Wiggins, 1994; Haller and Poje, 1998; Haller and Yuan, 2000). These methods are deterministic in that they rely on the full knowledge of the Eulerian flow field in order to derive the Lagrangian structures. However, observing the oceanic flow field in full (all spatio-temporal degrees of freedom) is a daunting challenge (Sanford et al., 2011). Perhaps the most widely-available data sets with global coverage are gridded satellite altimetry products (Ducet et al., 2000), in which a geostrophic relation is used to convert sea surface heigh anomaly to surface velocity fields (Wunsch and Stammer, 1998). The impact of uncertainties arising from this simplification, as well as of the missing spatial and temporal motions between satellite tracks (Chavanne and Klein, 2010) on transport estimates concern the main motivations of the present study.

The second issue that makes transport prediction so challenging in the ocean is related to the wide range of spatial scales in the ocean. Unlike classical turbulence, where a cascade of scales is generated by various fluid instabilities during the break down and dissipation of a primary coherent structure, the ocean contains a whole range of dynamically-distinct coherent structures that are forced by multiple processes, while coexisting and interacting together. In particular, the upper ocean boundary layer is stirred from below by mesoscale eddies, contains fronts that are created by surface and riverine buoyancy fluxes, convective plumes with a diurnal cycle, Ekman spiral by wind forcing as well as Langmuir circulations and Stokes drift by the action of surface waves. A particular class of processes, so-called submesoscales, received significant attention since they fall in between the relatively better studied 2D-like mesoscale motions and classical 3D turbulence (Müller et al., 2005). Submesoscale motions are defined as those having scales below the deformation radius, ranging from about tens of $\mathrm{km}$ down to the typical depth of the mixed layer, on the order of $100 \mathrm{~m}$, for the dynamics of which, the effect of Earth's rotation is significant (McWilliams, 2008). Submesoscales have been initially explored on the basis of theoretical and modeling stud- 
ies (Boccaletti et al., 2007; Thomas et al., 2008; Capet et al., 2008; Klein and Lapeyre, 2009; Taylor and Ferrari, 2010; Mensa et al., 2013; Sasaki et al., 2014), and are generally thought of as motions generated by frontogenesis and mixed-layer instabilities (Boccaletti et al., 2007; Fox-Kemper et al., 2008). A recent field exploration revealed the existence of submesoscale flows that are intensified in the upper ocean (Shcherbina et al., 2013). The question of whether submesoscales contribute significantly to surface material transport is studied in numerical models using relative dispersion metrics (Haza et al., 2008; Poje et al., 2010; Özgökmen et al., 2011; Özgökmen and Fischer, 2012). The primary findings from these studies is that it is still quite challenging to fit two distinct scales of motion, mesoscales and submesoscales, in numerical models due to computational expense, and that ocean experiments with a large number of drifters would be the only reliable approach to find the truth. An ocean experiment was conducted using 300 surface drifters, the Grand Lagrangian Deployment (GLAD), to verify or falsify the trends from modeling studies. Poje et al. (2014) concluded that submesoscales indeed contribute to relative dispersion near the Deepwater Horizon oil spill site. Nevertheless, the study by Poje et al. (2014) did not identify the exact mechanisms responsible for submesoscale dispersion.

The third complication for Lagrangian prediction in the ocean concerns the existence of strong vertical velocities acting on buoyant tracers. As initially shown by Zhong et al. (2012), and further studied by Huntley et al. (2015) and (Jacobs et al., 2015), convergence zones in the upper ocean create clustering of surface material. These convergence zones are linked to ageostrophic processes, indicating that geostrophic velocity fields (lacking vertical velocity component) cannot produce a realistic tracer distribution. Thereby the question arises about the errors in transport produced by missing scales of motion.

The main concept that truncating the spectrum of Eulerian motions can lead to differences in Lagrangian transport is not new, but was recognized by Griffa et al. (2004) in the context of a simple quasi-geostrophic model. Poje et al. (2010) analyzed a hierarchy of modeling approaches in order to gain more perspective on the matter, and concluded that relative dispersion is significantly impacted when both spatial and temporal scales of motion are truncated. Haza et al. (2012) developed and tested stochastic Lagrangian models to incorporate the effect of missing scales of motion on relative dispersion.

While the effect of submesoscales is highlighted by several metrics, in particular scaledependent relative dispersion, it is not yet clear whether submesoscales have any effect on transport. Conceptually, smaller scale turbulent features are usually imbedded within larger scale turbulent features, and smaller ones are transported by the large ones, as opposed to following a completely different path. This general concept is in fact fundamental to the development of subgrid-scale models in the large-eddy-simulation community (Sagaut, 2006) in the context of homogeneous 3D turbulence. It remains unclear to which extent it 
is applicable in oceanic multi-scale flows.

One of the ways to address this question is to compare in-situ measurements of transport pathways and those derived from satellite altimeters, or data-assimilating ocean models. Such comprehensive data sets are rare, but few have been available recently. For instance, during the DwH event, Olascoaga and Haller (2012) were able to predict the main pathways of the oil spill on the basis of LCS derived from an ocean circulation model that resolved mostly the mesoscale currents. Olascoaga et al. (2013) concluded that LCS from geostrophic velocities derived from altimetry data imposed an important constraint to the motion of drifters from the Grand Lagrangian Deployment (GLAD) in the Gulf of Mexico. A similar conclusion is also reached during a smaller drifter deployment in response to another oil spill in the Gulf of Mexico (Romero et al., 2015). A re-analysis of the GLAD data set by Beron-Vera and LaCasce (2015) using other metrics than scale-dependent relative dispersion and with the help of a high-resolution ocean model further emphasizes the importance of mesoscale control over transport. Nevertheless, Berta et al. (2015) found significant differences between the reconstructed Eulerian flow fields from GLAD and those derived from altimetric geostrophic fields.These differences are especially pronounced within the DeSoto Canyon region in the northern Gulf of Mexico, the site of the DwH spill, while the agreement between satellite-derived and drifter-derived surface velocity field is quite good in the interior, deep Gulf. Berta et al. (2015) attributed deviations from geostrophic currents to possible presence of river discharges, upwelling events along the continental shelf, wind-forcing diurnal or faster times scales, as well as submesoscale dispersion identified by Poje et al. (2014). Conclusions by Berta et al. (2015) are also consistent with d'Ovidio et al. (2015), who validated transport pathways derived from altimetry against trajectories of more than a hundred drifters and chlorophyll images using global, regional, and Ekman-corrected altimetry data. They found that while the Ekman-corrected product was the most reliable, differences at the meso- and smaller scales were evident as well. Clearly, the issue of whether and how submesoscales influence transport is complex, perhaps because it is rather subtle, cannot be generalized easily, and therefore, deserves continued investigation.

In this study, we aim to make progress in addressing the following question:

- Are LCS derived from geostrophic currents robust barriers to transport? That is to say, do they leak under submesoscale action? If there is a leakage, how much is the amount of material that gets across?

This problem is approached by focussing on a single coherent structure, a mesoscale eddy, evolving in a realistic ocean circulation modeled in the Gulf of Mexico. We prefer an elliptic structure for two main reasons. First, it is thought to have a capacity to contain and transport, or alternatively, to be totally shielded from the entry of large amounts of tracer. Second, submesoscale instabilities on the perimeter of a mesoscale eddy have been 
encountered in nature recently (Özgökmen et al., 2012). The superimposition of two distinct scales of instability poses a well-defined setting regarding the goals of the paper. We study the output from a high-resolution OGCM, in which a mesoscale eddy containing submesoscale perturbations along its rim is identified. The full fields are then spatially smoothed using different filters and Lagrangian characteristics of these different flows fields are compared in order to probe the question whether submesoscales have any effect on the overall tracer transport.

The paper is organized as follows: in Section 2, we detail the OGCM used and spatial filtering methods. The results are presented in Section 3. We conclude and point to future directions in Section 4.

\section{Methodology}

\subsection{Hydrodynamic model}

HYbrid Coordinate Ocean Model (HYCOM) is configured for the entire Gulf of Mexico. HYCOM uses isopycnal coordinates in the deep stratified ocean, pressure coordinates in unstratified regions including the mixed layer, and terrain-following coordinates in shallow coastal regions. The simulation has 20 layers in the vertical and $1 / 100^{\circ}$ horizontal resolution. The flow is forced by six-hourly Navy Operational Global Atmospheric Prediction System (NOGAPS) surface winds and heat fluxes. Cyclic boundary conditions south of the Yucatan Channel and in the Florida Straits are provided by a synoptically-forced 1/12 ${ }^{\circ}$ Atlantic HYCOM configured for much of the Atlantic Ocean, from $30^{\circ} \mathrm{S}$ to $70^{\circ} \mathrm{N}$ (Chassignet et al., 2007). The results from this larger domain simulation are obtained for years 1999-2002 and binned into monthly values to create a perpetual forcing and boundary conditions for the Gulf of Mexico inner domain. The model topography is derived from the two-minute NAVO/NRL DBDB2 global data set with true coastline and a minimum depth of $2 \mathrm{~m}$. Rivers are represented by point sources forced towards monthly climatological freshwater inflows. Further details of the model configuration can be found in Prasad and Hogan (2007). The k-Profile parameterization (KPP; Large et al. (1994)) is used to invoke vertical mixing. The model was run over 2010 and demonstrates dynamically-realistic behavior. In particular, the simulation generates a number of cyclones that tend to orbit around the anticyclonic Loop Current Eddies. The cyclones and anticyclones seldom cross the shelf break front in the northern Gulf of Mexico, although filaments associated with baroclinically-unstable eddies do generate cross-shelf exchange and interact with largely barotropic flows that tend to follow the shelf break front. Loop Current eddy shedding and wind-driven upwelling near the Yucatan Peninsula seem to be generally consistent with observations (Morey et al., 
2003; Walker et al., 2005; Sturges et al., 2005), although they occur non-contemporaneously because of lack of data assimilation.

The primary features that we are interested in this HYCOM simulation are that: (a) the model has enough numerical resolution to capture some of the submesoscale mixed layer instabilities, and (b) the flow field contains one of the so-called "Star Eddies" (Özgökmen et al., 2012) to allow us to estimate the influence of submesoscales on surface material fluxes across mesoscale material boundaries in elliptic coherent features.

\subsection{Spatial filtering}

The modeled submesoscale-permitting fields are spatially filtered in order to identify mesoscale transport barriers. The spatial filtering corresponds to the convolution operation

$$
u_{F}(\mathbf{x}, t)=G * u=\iint G(\mathbf{x}-\zeta) u(\zeta, t) d \zeta^{2},
$$

where $u$ is the full field, $u_{F}$ is the filtered field, $G$ is a filter function applied in the horizontal directions represented by the dummy variable $\zeta$.

Two filter functions are used for different purposes. The first is the Lanczos filter $G_{1}$, which can be expressed as:

$$
G_{1}(\delta)=\sin C\left(k_{C} \delta\right) \sin C\left(\frac{k_{C} \delta}{a}\right),
$$

where $a \geq 1$ is an integer (here, $a=2$ ), $k_{C}$ is the wavenumber cutoff, and $\delta$ is the distance from the location where the filter is applied. The filtered velocity at location $x_{0}$ becomes:

$$
u_{F}\left(x_{0}\right)=G_{1} * u\left(x_{0}\right)=\frac{\sum_{x=x_{0}-a \lambda_{C} / 2}^{x=x_{0}+a \lambda_{C} / 2} G_{1}\left(x-x_{0}\right) \cdot u(x)}{\sum_{x=x_{0}-a \lambda_{C} / 2}^{x=x_{0}+a \lambda_{C} / 2} G_{1}\left(x-x_{0}\right)} .
$$

The advantage of the Lanczos filter is that it establishes a clear cut off along the kinetic energy spectrum for wavenumbers $k>\delta^{-1}$, without affecting the kinetic energy for wavenumbers $k<\delta^{-1}$. Therefore, the Lanczos filter is ideally suited to separate submesoscales from mesoscales, as already pointed out in Mensa et al. (2013).

In order to simulate the geostrophic velocities as a proxy for current-generation altimeter measurements, a Gauss filter $G_{2}$ is also used:

$$
G_{2}(\delta)=\exp \left(-\frac{\delta^{2}}{2 \sigma^{2}}\right)
$$

where $\sigma$ is the width of the Gauss function. The value of $\sigma$ sets the filter strength to obtain similar average levels of strain and shear as the those of the AVISO altimetry product (cf section 3.4). 
Using $\sigma=20 \mathrm{~km}$, this filter is applied to HYCOM sea surface height (SSH) field $\eta$ as follows

$$
\eta_{F}\left(x_{0}\right)=G_{2} * \eta\left(x_{0}\right)=\frac{\sum_{x=x_{0}-3 \sigma}^{x=x_{0}+3 \sigma} G_{2}\left(x-x_{0}\right) \cdot \eta(x)}{\sum_{x=x_{0}-3 \sigma}^{x=x_{0}+3 \sigma} G_{2}\left(x-x_{0}\right)} .
$$

and the geostrophic velocities $\mathbf{V}_{G}$ are then calculated from:

$$
\mathbf{V}_{G}=-\frac{g}{f} \mathbf{k} \times \nabla \eta_{F}=\left(\begin{array}{c}
-\frac{g}{f} \frac{\partial \eta_{F}}{\partial y} \\
\frac{g}{f} \frac{\partial \eta_{F}}{\partial x}
\end{array}\right)
$$

where $g$ is the gravity constant, $f$ is the Coriolis parameter, and $\mathbf{k}$ is the unity vector in the vertical axis.

\subsection{Gridded altimetric data}

Data from AVISO (Archiving, Validation and Interpretation of Satellite Oceanographic data) is also employed as a reference surface velocity field. The geostrophic velocity field is assumed to be of the form $v(x, t)=g f\left(x_{2}\right)^{-1} \nabla^{\perp} \eta(x, t)+\nabla \varphi(x, t)$, where $g$ is the acceleration of gravity; $f\left(x_{2}\right)$ is the latitude-dependent Coriolis parameter; $\eta(x, t)$ is sea surface height anomaly from AVISO; and $\varphi(x, t)$ is such that the normal projection of $v(x, t)$ at the coastline vanishes (Marshall et al., 2006). The steady $\eta(x, t)$ component is given by a mean dynamic topography constructed from altimetry data, in-situ measurements, and a geoid model (Rio and Hernandez, 2004) while the transient component is given by gridded altimetric SSH anomaly measurements provided weekly at $0.25^{\circ}$-resolution (Le Traon et al., 1998). Jason-1 and 2, and Cryosat-2 traverse the Gulf of Mexico about 10 times on average per week.

\section{Results}

\subsection{Description of the flow field}

We define as "Star Eddy" a mesoscale eddy subject to sinusoidal perturbations along its rim. These patterns give it the appearance of a star (cf Fig. 1), hence the name. The perturbations are likely to be associated with the baroclinic instability of the Eddy's density front within the mixed layer. In other words, since the radius of deformation within the surface mixed layer is smaller than at depth, two scales of motion are superimposed and submesoscale features are embedded within and transported by the mesoscale flow. A Star Eddy constitutes an excellent test-bed to study the effect of disparate scales of flow on tracer transport. 
Similar multi-scale flows were studied in Özgökmen et al. (2012) by computation. Following Özgökmen et al. (2012), the radius of deformation in the mixed layer is $R_{d}^{M L}=$ $\sqrt{\frac{g}{\rho_{0}} \Delta \rho_{M L}^{\prime} h_{0}} / f$, where $h_{0}$ is the mixed layer depth, $\Delta \rho_{M L}^{\prime}$ is the density change across the surface front, $g=9.81 \mathrm{~m} / \mathrm{s}^{2}$ is the gravitational acceleration, $\rho_{0}=1026 \mathrm{~kg} / \mathrm{m}^{3}$ is seawater density, and $f=6.5 \times 10^{-5} \mathrm{~s}^{-1}$ is the Coriolis frequency at $26.5^{\circ} \mathrm{N}$. The radius of deformation below the mixed layer is $R_{d}=\sqrt{\frac{g}{\rho_{0}}\left(\Delta \rho^{\prime}-\Delta \rho_{M L}^{\prime}\right)\left(H_{0}-h_{0}\right)} / f$, where $\Delta \rho^{\prime}$ is the change across the depth of the mesoscale eddy. Estimating $\Delta \rho_{M L}^{\prime}=0.038 \mathrm{~kg} / \mathrm{m}^{3}, h_{0}=150 \mathrm{~m}$, $H_{0}=400 \mathrm{~m}, \Delta \rho_{M L}^{\prime}=2.085 \mathrm{~kg} / \mathrm{m}^{3}$ from the model fields, one gets $R_{d}^{M L}=3.6 \mathrm{~km}$ and $R_{d}=34.1 \mathrm{~km}$. Thus, $R_{d} / R_{d}^{M L} \approx 10$, and as per Olson (1991), oceanic eddies have a radius that is 2-4 times larger than $R_{d}$. These estimates are consistent with what is seen in Fig. $1 \mathrm{~b}$.

In order to visualize transport patterns associated with the Star Eddy, a tracer patch consisting of 1.2 million passive surface particles is launched uniformly on a square encompassing the Eddy at time=15 days (corresponding to January 15, 2010). Fig. 2a illustrates the patchiness of the tracer distribution a month after the launch. It shows that the tracer concentration is no longer homogeneous in this setting. As indicated by satellite images of sargassum and green algae (Fig. 2b,c), similar distributions are observed in the presence of buoyant tracers in the real ocean as well. This result was already put forward by Zhong et al. (2012), who illustrated that the patchiness is due to ageostrophic submesoscale instabilities that cause horizontal convergence and divergence in the surface flow field. At the same time, one can observe tracer filaments spiraling from the core of the Eddy, in a pattern that seem to be dominated by mesoscale motions. The question is therefore whether mesoscales control the entire tracer distribution, or submesoscale motions have a tangible effect on tracer transport in and out of the Eddy as well.

\subsection{Tracer distribution with Lanczos filter}

Three different parameter gains for filter $G_{1}$ are applied to the surface velocity field in order to monitor the effects of a gradual elimination of submesoscale motions. These correspond to the cutoff wavelengths $\lambda_{C}=2 \pi / k_{C}=20,40$, and $80 \mathrm{~km}$. The impact of filter parameter changes on the kinetic energy wavenumber spectrum is illustrated in Fig. 3; the filter removes most of the kinetic energy at the wavelengths below $\lambda_{C}$. At the range $\lambda>\lambda_{C}$, the kinetic energy is preserved. In order to provide a more physical perspective, the resulting surface speed fields are shown in Fig. 4; Lancsoz filter gradually removes submesoscales in the flow field without changing the speed of the larger scale features. Another important consequence of the effects of submesoscales is emphasized in Fig. 5. As submesoscales are filtered out by $G_{1}$, horizontal divergence, initially on the order of the local Coriolis frequency $f$, is removed as well. Horizontal divergence on the order of $f$ is particularly pronounced around the periphery 
of the Star Eddy, indicating that these sinusoidal modes involve significant vertical motion, as one would expect from submesoscale motions associated with fronts (McWilliams, 2008). Overall, we find that using $G_{1}$, the mesoscale features and their magnitudes are preserved. Thus a comparison of tracer evolution can isolate the submesoscale contribution to transport.

The effect of filtering on dispersion is quantified using the scale-dependent Finite Scale Lyapunov Exponent (FSLE), $\lambda(\delta)$. The FSLE is a diagnostic closely related to relative dispersion (Artale et al., 1997; Aurell et al., 1997)

$$
\lambda(\delta)=\frac{\log (\alpha)}{\langle\tau(\delta)\rangle}, \quad \text { with } \quad 1<\alpha<2
$$

where $\langle\tau(\delta)\rangle$ is the averaged time taken by all particle pairs to separate from the distance $\delta$ to $\alpha \delta$. The FSLE has been widely used in order to investigate the influence of different types of spatial and temporal filtering on material dispersion (Haza et al., 2008; Poje et al., 2010; Haza et al., 2012). Here, results are shown separately for $\delta<20 \mathrm{~km}$, for which original pairs are more suitable for the statistics (Fig. 6a), and for $\delta>20 \mathrm{~km}$, for which chance pairs provide higher density data (Fig. 6b), as per sensitivity studies conducted in Haza et al. (2010). One can see that the $G_{1}$ filter preserves the FSLE at scales $\delta>20 \mathrm{~km}$, independently of the cutoff wavelength. For this scale range, dispersion, including the result from gridded altimeter data, is consistent with Richardson's scaling, $\lambda \sim \delta^{-2 / 3}$. For $\delta<$ $20 \mathrm{~km}$, or in the submesoscale regime, we find a gradual reduction of the maximum FSLE with the increasing amplitude of $\lambda_{C}$, in that the FSLE plateau becomes wider, applying to a larger range of scales. This means that as submesoscales are filtered from the flow field, maximum dispersion rate is also reduced. This is consistent with modeling results shown in Poje et al. (2010), as well as the observational findings in the Gulf of Mexico (Poje et al., 2014). Overall, this is reassuring that the $G_{1}$ filter is working quite well by gradually removing scales of motion as dictated by $\lambda_{C}$, and their dispersive effect on particle motion. Thereby, filtering with $G_{1}$ provides a good tool for the problem of interest here, which is submesoscale-induced transport across mesoscale material boundaries.

Tracer evolutions with velocity fields filtered with different $\lambda_{C}$ of $G_{1}$ are plotted 11, 31 and 51 days later in Figs. 7, 8 and 9, respectively. Two main characteristics emerge from these plots. The first is that the patchiness is substantially reduced by filtering. This is consistent with the dramatic reduction in flow divergence, even with the weakest $\left(\lambda_{C}=20 \mathrm{~km}\right)$ filter. For the strongest filter $\left(\lambda_{C}=80 \mathrm{~km}\right)$, the patchiness disappears completely. It confirms that the horizontal divergence is the main cause of particle concentration patchiness. The second observation is that the overall large-scale pattern of the tracer remains mostly unchanged.

Why the larger-scale transport pattern doesn't change with $G_{1}$ filtering can be explained by the LCS shape. The LCSs are considered to represent transport barriers and approxi- 
mated by maps of local FSLE as follows:

$$
\lambda\left(i, j, d_{i}, d_{f}\right)=\frac{\log \left(d_{f} / d_{i}\right)}{\tau},
$$

where $\tau$ is the time it takes a pair of particles $d_{i}$ apart at location $(i, j)$ to separate to the distance $d_{f}$. The repelling LCS correspond to the FSLE extrema or ridges computed forward in time, while the attracting LCS are obtained from computing the FSLE backward in time. Note that the local FSLE and scale-dependent FSLE are two different metrics even if they have the same name (the latter computes statistical values of dispersion by varying the initial distance $\delta$, while the former computes a local rate of dispersion for a fixed initial distance $\left.\delta_{i}\right)$.

LCS maps are illustrated in Fig. 10. The LCS associated with the full field is quite complicated. In particular, the Star Eddy has very complex LCS around its periphery while the elliptic interior $G_{1}$ filter removes the small scale dispersive features. However, filtering does not alter the large-scale LCSs. As a result, the tracer is steered, stretched and deformed according to the attracting and repelling LCSs of the mesoscale flow features. The impact of spatial filters on the LCS field was already explored in Haza et al. (2008), where it was found that up to a certain filter strength, the time-dependent component of the entangled Lagrangian structures is maintained, even if the intensity of the local velocity gradients is reduced. Similarly, the impact on the scale-dependent relative dispersion is preserved at the meso and larger scales as long as the low-pass filter does not remove the mesoscale features (Poje et al., 2010).

For the rest of this manuscript LCS is referred to transport barriers computed using the FSLE ridges from the filtered (mesoscale) field. As it will be shown below, the accuracy of the FSLE calculation is confirmed by superimposing particle advection using the filtered fields. Thus methodological errors for identifying the exact LCS line is negligible with respect to dynamical differences between the mesoscale-only and full fields.

\subsection{Material leakage across mesoscale LCS}

These results have far-reaching implications for a variety of practical applications in terms of Lagrangian transport. In particular, the following question arises:

- If one relies on mainly a mesoscale field constrained by synoptic observations such as gridded altimetric SSH anomaly, how robust are the Lagrangian transport pathways in the presence of submesoscales?

In an attempt to explore this issue further, we launch a smaller tracer patch on the inner edge of the Star Eddy's main attracting LCS, which is delineating the edge of the eddy (as 
defined by the filtered field). We pick the strongest gain $\lambda_{C}=80 \mathrm{~km}$ for the $G_{1}$ filter so that all submesoscales are filtered out and only the mesoscales remain. The tracer is then advected by both the full flow and the filtered flow, and its time evolution is displayed in Fig. 11. One can see that in spite of similar large-scale patterns, the tracers end up having different distributions. The tracer in red is advected by the filtered velocities and its evolution is consistent with the plotted LCS field, that is, some time after the launch, it stretches and molds itself along the nearby attracting LCS. As stated above, this figure clearly shows the consistency of the computed LCS lines and particle motion, thereby removing the possibility of methodological errors in causing leakage across these mesoscale barriers. On the other hand, the blue tracer is advected by the full field. It appears to be steered in the general direction of the filtered flow, while simultaneously tracer filaments are seen to move across the LCS, effectively diverging from the red tracer. Note that for day $>45$, portions of the blue tracer are steered by other LCS than those dictating the motion of the red tracer.

We note that while crossing the mesoscale LCS barrier by submesoscale leakage is quite fast (days), the leaked tracer remains captive to the vicinity of the LCS for much longer. It seems that these long times scales can be partly explained by the idealized submesoscalemesoscale coupling results by Ramachandran et al. (2014), who have shown how fronts confined to the mixed layer and those extending below it can evolve differently over timescales considerably longer than that describing mixed-layer submesoscale instabilities alone. One primary difference here is that unlike in Ramachandran et al. (2014), where a single mesoscale front is simulated, the Star Eddy in HYCOM is imbedded in and surrounded by numerous mesoscale features. The difference between tracers from the full and filtered flows starts growing when the leaked tracer leaves the influence of the initial transport barriers and gets attracted to another one.

From these simulations, one can deduce that in the presence of submesoscales, a tracer can leak through the mesoscale LCS, even though it is steered in the general direction of the mesoscale flows. Let's remind that the displacement of a fluid parcel is supposed to be fully constrained by the transport barriers. Any leakage across the LCS transport barriers puts the robustness and meaning of these LCS in question. The LCS field associated with mesoscale flows is no longer robust in the presence of baroclinic instabilities arising from submesoscale flows, even if mesoscale LCS still have a significant control over the pathways. This conclusion brings us to the next logical question:

- How much do the submesoscale flows alter Lagrangian transport compared to mesoscale flows? The deviation of the actual transport with respect to mesoscale pathways needs to be quantified.

How to quantify the tracer leakage is not a trivial problem for two main reasons. First, particles crossing a barrier and moving away from the Eddy can later move back towards 
the Eddy by crossing another barrier. Second, if a portion of tracer moves toward the center while its filtered counterpart remains on the inner edge of the eddy, does it count as leaking?

One needs to understand first how the submesoscales resolved in HYCOM end up altering the original tracer distribution to this extent. What appears to increase dramatically the tracer dispersion is the combination of transport barrier crossing and barrier steering. Particles crossing a barrier are seen in Fig. 11 to be later attracted to a nearby barrier. Indeed, the mesoscale LCS still exert a dominant constraint on the Lagrangian transport. However, the simple process of moving from one LCS ridge to another, results in a fast divergence from the original path taken by the same particle, advected solely by the mesoscale field. For instance, consider a particle-pair, where one particle crosses a barrier while the other doesn't. After some time, the dispersion of the pair is substantially higher than one where both particles are fully constrained by the mesoscale LCS.

In this context, the potential of leaking at the edges of the LCS is what defines the departure from pure mesoscale Lagrangian transport, and provides a viable alternative for the quantification of the submesoscale contribution. And while deviations from the mesoscale flow at the location of the attractive LCS are indication of barrier crossing, the amount of leaked tracer resulting in a higher dispersion can be identified by the additional spatial coverage of the transport barriers by the tracer.

There are then two main metrics we consider: First, we estimate average flow discrepancies in the immediate vicinity of the mesoscale LCS. Second, we quantify the portion of the full-flow tracer hitting the mesoscale LCS that are unoccupied by the filtered counterpart, and corresponds therefore to a lower bound estimate of the leaking portion.

Note that the direct comparison of the instantaneous velocity fields at the transport barriers introduces several biases, because the temporal and spatial resolutions of the lowpassed and full velocity fields in a realistic context are not the same. In this case, HYCOM outputs have a 3 hour interval for a $1 \mathrm{~km}$ resolution, whereas a daily output interval is used for the low-passed fields, and the effective spatial resolution is much coarser. The mesoscale FSLE ridges are also computed only once a day, to be more representative of a realistic scenario. Therefore, calculating the daily instantaneous flow discrepancy in the Eulerian framework would not make much sense, since it doesn't take into account the spatiotemporal variability of the submesoscale motions. Preliminary tests have yielded much larger discrepancies in the flow fields with the Eulerian approach. The velocity fields generally differ less with time-averaging. In this method, the mean Lagrangian velocities are used based on the initial and final positions of the particles in a 1-day interval. This way, the averaged velocities reflect the cumulative time-integration of the particles advection subject to several submesoscale events of shorter time-scales. 
Once the particle-based tracer advected by the low-passed velocity field is molded along its corresponding attractive LCS, Lagrangian velocities are estimated for a subset of particles based on two consecutive days, and denoted as $\mathbf{V}_{L P}\left(p_{i}\right)$, where $p_{i}$ is the $i^{t h}$ particle.

$\mathbf{V}_{L P}\left(p_{i}\right)$ corresponds to the Lagrangian velocity of a one day particle motion starting at the transport barrier.

Regarding the tracer advected by the full field, the spatial variability and higher resolution introduce an additional bias to the velocity comparison. For this reason, the velocities of particles within a radius of few $\mathrm{km}$ are averaged as well, before be compared to the Lagrangian velocities of the low-passed field. It is one way to bring back the full field advection to one of coarser spatio-temporal resolution for consistency. The Lagrangian velocity counterpart for the full flow at the same location is estimated by computing the averaged velocity of particles within a $5 \mathrm{~km}$ radius of $p_{i}$, denoted as $\mathbf{V}_{1 \mathrm{~km}}\left(p_{i}\right)$. This distance was picked sufficiently small to avoid overlapping different LCS ridges. The difference in flow direction $\Delta \Theta=$ $\Theta(f u l l)-\Theta(L P)$ is the angle between $\mathbf{V}_{1 k m}\left(p_{i}\right)$ and $\mathbf{V}_{L P}\left(p_{i}\right)$.

The velocity component of $\mathbf{V}_{1 k m}\left(p_{i}\right)$ projected on $\mathbf{V}_{L P}\left(p_{i}\right)$ at the location of $p_{i}$ is:

$$
\mathbf{V}_{\text {proj }}\left(p_{i}\right)=\frac{\mathbf{V}_{1 k m}\left(p_{i}\right) \cdot \mathbf{V}_{L P}\left(p_{i}\right)}{\mathbf{V}_{L P}\left(p_{i}\right) \cdot \mathbf{V}_{L P}\left(p_{i}\right)} \mathbf{V}_{L P}\left(p_{i}\right),
$$

and the velocity component normal to $\mathbf{V}_{L P}\left(p_{i}\right)$ or vector rejection becomes

$$
\mathbf{V}_{r e j}\left(p_{i}\right)=\mathbf{V}_{1 k m}\left(p_{i}\right)-\mathbf{V}_{\text {proj }}\left(p_{i}\right) .
$$

Note that $\mathbf{V}_{r e j}\left(p_{i}\right)$ is the velocity component leading to LCS barrier crossing. This projection is equivalent to a rotation of $\mathbf{V}_{1 \mathrm{~km}}\left(p_{i}\right)$ from the cartesian coordinates to the LCS coordinates.

Fig. 12 illustrates the method described above by plotting the tracer at day 55, or some 40 days after it was launched on the inner edge of the Star Eddy's main LCS and advected by the Lanczos $\lambda_{C}=80 \mathrm{~km}$ filtered velocities. The red vectors mark the direction of the tracer, corresponding also to the displacement of the LCS. The blue vectors are the velocities of the full flow tracer, while the green vectors are the velocity components normal to the filtered velocities (vector rejection), i.e. moving perpendicular to the flow along the attracting LCS. The difference in flow direction along the LCS is displayed in the bottom panel, and shows a small average discrepancy $\left(4^{\circ}\right)$, but with a high variability $\left(51^{\circ}\right)$ in this case. Values for other days and filters are listed in Table 1 . This result means that particles do have the potential to leak, even if they are steered by the mesoscales. The vector rejection indicates that on average $7 \%$ of the total velocities are perpendicular to the steering velocities, confirming that most of the motion is still along the LCS.

Next, we take a closer look at the particle leakage across the LCS. The mesoscale LCS are digitized on a $1 \times 1 \mathrm{~km}$ grid after setting a threshold for the local FSLE maxima. For a given day after the tracer release, the amount of tracer having crossed the LCS barriers is 
computed by counting the total number of particles within a $5 \mathrm{~km}$ radius of a digitized LCS that is not presently occupied by the tracer advected by the low-passed velocities. The same experiment is plotted differently in Fig. 13 every 10 days. The tracer advected by using G1 filter with $\lambda_{C}=80 \mathrm{~km}$ velocities is colored in red, while the full tracer is represented in blue and green. The green color corresponds to particles in the vicinity (within a distance of 5 $\mathrm{km}$ ) of a mesoscale LCS not occupied by the tracer of the filtered flow, hence to particles that have crossed at one time the mesoscale transport barriers. We can see that most of the green tracer is located on the outer edge of the Star Eddy, where submesoscales tend to develop. In time, the escaped particles move on to other attracting LCS in the periphery, where they are corralled. Some eventually cross the LCS.

The same experiment is repeated for the other filter strengths and different times, and the results of both metrics are summarized in Fig. 14 and Fig. 15. Fig. 14 displays the time series of the mean angle discrepancy and standard deviation on the LCS of the filtered fields. The differences are not significant, except perhaps a month after the launch in the std of $\Delta \theta$ between $\lambda_{C}=80 \mathrm{~km}$ and the weaker filter cases: the standard deviations using $\lambda_{C}=20 \mathrm{~km}$ and $\lambda_{C}=40 \mathrm{~km}$ are a bit lower, as one would expect from a velocity field closer to the full field. Note also from Table 1 that the vector rejection remains at similar low percentages, which confirms that the differences in flow direction is not much dependent on the filter strength.

On the other hand, marked differences appear clearly in the percentage of leaked particles (Fig. 15) The estimated portion of leaking tracer is higher close to the launch date. It must have to do with the fact that the tracer has not dispersed yet, so that big chunks of tracer escape (cf top left panel of Fig. 13) whenever a frontal instability disrupts the main transport barriers. Later on, some of the particles that have escaped the eddy reenter it. This behavior is confirmed by other tests (not shown here). It also explains why all three experiments lead to a decrease after 15-20 days. About a month later, the portion of leaked tracer is about $20 \%$ for $\lambda_{C}=80 \mathrm{~km}$ versus $5-10 \%$ for the weaker filters. Here again, the closer similarity of the weak filters to the true fields means that more leaked particles are later recovered by the LCS, and/or the particles recross those barriers.

We have also released tracers in the full and filtered fields in an eddy identified from HYCOM in the summer time. Seasonality of the submesoscales in HYCOM was documented by Mensa et al. (2013) in that submesoscale instabilities are stronger in winter than in summer. Summer eddies are virtually free from the sinusoidal perturbations around the rim. The turn over time scale of the summer eddy is approximately 3 days in comparison to 11-15 days for the Star Eddy. The tracers are advected for 57 days and the computed leakage is found to be only $2 \%$. 
While the LCS associated with the mesoscale features (using $\lambda_{C}=80 \mathrm{~km}$ ) set the main flow pathways, the material leakage, some $20 \%$ of the tracer through the mesoscale transport barriers seems quite significant.

\subsection{Tracer results from altimetry proxy}

As illustrated by the wavenumber kinetic energy spectrum (Fig. 3), a wider range of wave numbers is affected by the $G_{2}$ filter. A portion of the kinetic energy from the large wavelengths is removed with the totality of the small wavelengths, and resulting in an alteration of the mesoscale momentum. The differences between both filters are seen in the resulting velocity magnitudes of Fig. 4: The Lanczos filter preserves the momentum of the main features, such as the Loop Current and the Star Eddy. But the geostrophic velocities from the low-passed SSH fields display smoother fields with lower kinetic energy levels for all mesoscale features.

Altimetry products require several spatial and temporal interpolations to convert satellite track observations into a time-series of SSH values on a regular grid. This process is done at the expense of the kinetic energy and strain fields which leads to excessively smooth geostrophic velocities. The effect of the spatiotemporal interpolations on the altimetry data is mimicked in this study by a Gauss filter applied to the model SSH field. Its impact on the geostrophic velocity product is illustrated by the kinetic energy spectrum plotted in red in Fig. 3, and by the velocity magnitudes in Fig. 4. One can see that the mesoscale features are smeared when compared to the $80 \mathrm{~km} \lambda_{c}$ case, and their magnitudes are reduced. This means that unlike the Lanczos experiments where only the submesoscale fields were affected, the mesoscale features have lost some of their momentum. Regarding the degree of realism of this filtering process, we refer to the AVISO product in terms of scale-dependent FSLE (or their impact on the rates of relative dispersion at all scales). As illustrated in Fig. 6, the smoothing effect is represented by the maximum Lyapunov exponent computed from original pairs (upper panel), and shows that the $G_{2}$ filtered sea surface height and resulting geostrophic velocities of HYCOM successfully matched the averaged hyperbolicity of AVISO. The impact on the mesoscales is visible in the chance-pair computation (lower panel) from the slight reduction in $\lambda$, although the slope is the same. This is due to the reduction in the momentum of the mesoscale flow. We do not observe such a difference with AVISO in that the real altimetry product does a better job at preserving the relative dispersion of the large scales. It may be due to the less homogeneous characteristics of the spatio-temporal interpolations of the altimeter measurements. The mesoscale velocity magnitudes and shear must be higher along the track than they are in between tracks where interpolations are required. The scale-dependent FSLE is more biased toward regions of high strain. But overall, the similarities with AVISO are good enough for our purpose here. 
Fig. 16 shows the evolution of the particle-based tracer concentration when advected by the geostrophic velocities. Comparison with the $G_{1}$-filtered cases in Figs. 7 through 9 illustrates the changes in the large scale patterns of the tracer distribution. Most of the tracer is retained inside the eddy, even after 50 days. Here again, we look at the LCS field for confirmation. The attracting LCS of the altimetry proxy is plotted in the bottom panel of Fig. 17, as well as the LCS of the full field, and the Lanczos experiments using $\lambda_{C}=80$ $\mathrm{km}$. As expected, the FSLE ridges are modified by the filter in such a way as to increase the ellipticity of the Star Eddy by curving the LCS ridges (Haller and Beron-Vera, 2013). The comparison between the filtered fields using $\lambda_{C}=80 \mathrm{~km}$ and this case clearly illustrates the elliptical bias of the geostrophic velocities from today's altimetric fields.

The same launch described in Fig. 11 is now repeated with the altimetry proxy and plotted in Fig. 18. One can see that the red tracer advected by the $G_{2}$-filtered flow field does not evolve as fast as the counterpart using the $G_{1}$ filter with $\lambda_{C}=80$ on the attracting LCSs. As a result, the dispersion gap between the red and blue tracers is more pronounced. Fig. 15 shows a percentage of leaked particles at least twice as high as the Lanczos estimates, and going up to $50 \%$.

\subsection{Null-case: summer eddy}

A null-case to appreciate better the role of submesoscale features is provided by summer conditions. Seasonality of the submesoscales in HYCOM was documented by (Mensa et al., 2013) in that submesoscale instabilities are stronger in winter than in summer, mainly because of deeper mixed layers. We have released tracers in a summer time eddy in the same region from HYCOM (Fig. 19). The turn over time scale of this eddy is approximately 3 days in comparison to 11-15 days for the Star Eddy. The tracers are advected for 57 days and the computed leakage is $2 \%$, namely much less than for the case studied for the winter Star Eddy. Thus, this final numerical test further strengthens the conclusion of this study that it is the submesoscale motions that create the material leakage across mesoscale transport barriers.

\section{Conclusions}

The main purpose of this study is to investigate the role of submesoscale motions on Lagrangian transport. Many Lagrangian prediction studies depend on observational constraints of the mesoscale circulation from satellite altimetry products, either directly from the derived geostrophic velocities, or indirectly from their assimilation into ocean forecast models. In both cases, the scales of motion below the radius of deformation are not well captured at 
the present time. As recent studies reveal the existence of submesoscale flows in the upper ocean boundary layer, it is becoming important to quantify their contribution on Lagrangian transport. There have been studies showing the significance of submesoscales using scaledependent dispersion metrics, but in many applied problems, the central topic is Lagrangian transport, namely, where and how surface tracer travels? To provide an answer, one needs to superpose all the processes at different scales contributing to transport.

There is no doubt that the mesoscale circulation is a dominant steering mechanism. Yet, submesoscales and mesoscales operate in somewhat different ways, especially on buoyant tracers at the surface of the ocean. Here, we expand on the concept that the addition of an ageostrophic flow with non-zero convergence can modify to some degree the transport predictions relying solely on 2D quasi-geostrophic flows. We specifically focus on a case, where submesoscale motions emerging from surface mixed-layer frontal instabilities coincide spatially with mesoscale frontal dynamics, because their relative contributions to transport can be assessed.

The problem is studied using a HYCOM simulation of the Gulf of Mexico circulation with a horizontal resolution of $1 / 100^{\circ}$, capturing part of the submesoscale spectrum as well as the mesoscales, thereby allowing for multi-scales interactions. The analysis is entirely focused on an anticyclonic mesoscale eddy imbedded with submesoscale motions due to the deep winter mixed layer. Submesoscales are separated from the mesoscales by applying a Lanczos low-pass filter to the HYCOM surface velocity field. The distributions of particlebased tracers advected by both full and filtered flow fields were then compared in relation to the mesoscale transport barriers. Also an altimeter proxy was emulated given that altimeterderived geostrophic velocity fields are commonly used due to their global availability.

The following results are obtained on the basis of the Lanczos filter. We confirm the findings from previous studies that submesoscale-induced surface divergence field generates regions of high and low tracer concentrations, leading to a patchy tracer distribution. While this result is not new, it is important to show that it happens in yet one more model that is different from those used in previous works, and therefore, it is something robust with respect to model numerics and parameterizations. A patchy tracer distribution is an important consideration in practical applications, such as response efforts to oil spills, in which resources may be limited with respect to the size of the spill area. Encountering as much oil as possible during daylight hours is important. It is clearly shown that the submesoscales add a significant range of structure to the mesoscale LCS field. These submesoscale LCS are intertwined with the mesoscales, and in many cases, intersect them, thereby leading particles to cross the mesoscale transport barriers. Quantification of Lagrangian velocities along the mesoscale LCS indicates deviations in flow direction along the transport barriers with an average of $2^{\circ}$ to $15^{\circ}$, with standard deviations of up to $51^{\circ}$. The velocity norms 
contributing to barrier-crossing is found to be in the range of $5 \%$. These results confirm that the submesoscales can lead to deviations in the transport by mesoscales, while the steering remains mostly (95\%) set by the mesoscales.

The resulting interplay between the mesoscales and the submesoscales can be summarized as follows: the attracting LCSs of the mesoscales are still dominating particle advection. However, the submesoscales render the mesoscale-based transport barriers less effective, allowing particles to cross them. The process does not require a massive disruption of the mesoscale flow, as the submesoscales velocities are still an order of magnitude smaller. Subsequently, particles having crossed a mesoscale barrier are attracted to a nearby barrier, and drift following mostly the mesoscale flow. They may or may not cross that barrier, and so on. Therefore, we find that the submesoscale-induced transport leakage is a subtle process, yet it exists and is not negligible.

Based on these observations, a method is developed to calculate a lower-bound estimate for the amount of tracer crossing the mesoscale transport barriers. The leakage of tracer on a localized launch near the edge of the Star Eddy is found to be about $20 \%$ after a month of advection. Regarding the altimetry proxy, a Gaussian filter was applied to HYCOM's SSH field with a strength set to match the maximum hyperbolicity from the AVISO SSH geostrophic estimate. LCSs computed from these geostrophic velocities display signatures of a modified mesoscale flow, in which velocities are reduced, and there is a clear bias towards increased ellipticity of the Star Eddy. This is to say that, a tracer launch encompassing the Star Eddy using this flow field remains mostly contained within its main boundaries after a month, while the comparison with the full-field advection reveals that the proportion of the tracer leaking through the altimeter-like LCSs is $50 \%$.

Considering that a significant portion of the tracer has not been constrained by the various mesoscale LCSs derived from two types of spatial filtering, one can conclude that in the presence of submesoscale motions, the mesoscale LCSs are leaky transport barriers, which still exert a great degree of control over the upper ocean tracer transport. The apparent contradiction between the relatively small flow discrepancies at the transport barriers and the more substantial tracer leakage makes sense when one takes into account the interplay of mesoscale barrier crossing and barrier steering which contributes to a significant departure from Lagrangian transport predictions based solely on mesoscale flows.

A number of questions remain to be pursued in future studies. First there is the issue of spatio-temporal variability about the submesoscales. Mensa et al. (2013); Sasaki et al. (2014) have shown that submesoscales are most pronounced in the upper ocean boundary layer during winter time due to deeper mixed layer. This finding implies that summer time transport can still be dominated by mesoscale processes. Second, the issue of winter time forcing is nontrivial for surface transport since strong winds may wipe out all coherent 
structures and the transport could be entirely driven by wind and Stokes drift (Curcic et al., 2016). Third, the influence of waves on transport has not gathered much attention thus far, but in addition to surface waves, internal gravity wave signatures may leak into the upper ocean, especially during summer months when the mixed layer is shallower and the mixed layer base can sustain significant wave activity. Fourth, latitude dependence of coherent structures is another important factor to consider. Here we focus on the Gulf of Mexico, where mesoscales and submesoscales are quite distinct from one another since the former is set by the large radius of deformation of the deep circulation and the latter by the relatively thin upper ocean mixed layer. At higher-latitudes, such scale separation could be much smaller (due to smaller radius of deformation and deeper mixed layers) so that the distinction in transport may be negligible. Fifth is the issue of how to represent these transport processes when they are unresolved by OGCMs. Most parameterizations used in modeling community are Fickian. If the transport process does not exhibit a diffusive pattern of dispersion, such as clearly the case of patchiness within an elliptic structure, then there should be a recognition that diffusive subgrid-scale parameterizations will not work in models that do not have the resolution to capture submesoscale-induced transport. Perhaps resolving these smaller anisotropic non-diffusive coherent structures is the only accurate path forward.

Acknowledgements: This research was made possible with the support from the Office of Naval Research (Grant \#N000141110087) and the Gulf of Mexico Research Initiative. We thank F. d'Ovidio for insightful discussions. We are grateful to the two anonymous referees for many constructive suggestions, including the addition of the summer eddy test case, shown Fig. 20 of the manuscript.

\section{References}

Aref, H., 1984. Stirring by chaotic advection. J. Fluid Mech. 192, 115-173.

Artale, V., Boffetta, G., Celani, A., Cencini, M., Vulpiani, A., 1997. Dispersion of passive tracers in closed basins: Beyond the diffusion coefficient. Phys. Fluids 9, 3162-3171.

Aurell, E., Boffetta, G., Crisanti, A., Paladin, G., Vulpiani, A., 1997. Predictability in the large: an extension of the concept of Lyapunov exponent. J. Phys. A 30, 1-26.

Beron-Vera, F., LaCasce, J. H., 2015. Statistics of simulated and observed pair separation in the Gulf of Mexico. J. Phys. Oceanogr. submitted.

Berta, M., Griffa, A., Magaldi, M., Özgökmen, T. M., Poje, A. C., Haza, A. C., Olascoaga, 
J., 2015. Improved surface velocity and trajectory estimates in the Gulf of Mexico from blended satellite altimetry and drifter data. J. Atmos. Ocean. Technol. 32, 1880-1901.

Boccaletti, G., Ferrari, R., Fox-Kemper, B., 2007. Mixed layer instabilities and restratification. J. Phys. Oceanogr. 37, 2228-2250.

Broecker, W., Gerard, R., Ewing, M., Heezen, B., 1960. Natural radiocarbon in the Atlantic Ocean. J. Geophys. Res. 65/9, 2903-2931.

Capet, X., McWilliams, J., Molemaker, M., Shchepetkin, A., 2008. Mesoscale to submesoscale transition in the California Current System: I flow structure, eddy flux and observational tests. J. Phys. Oceanogr. 38, 29-43.

Chassignet, E. C., Hurlburt, H. E., Smedstad, O. M., Halliwell, G., P.J. Hogan, A. W., Baraille, R., Bleck, R., 2007. J. Mar. Res. 65, 60-83.

Chavanne, C., Klein, P., 2010. Can oceanic submesoscale processes be observed with satellite altimetry? Geophy. Res. Lett. 37, L22602.

Crone, T., Tolstoy, M., 2010. Magnitude of the 2010 Gulf of Mexico oil leak. Science 330, 634.

Curcic, M., Chen, S., Özgökmen, T. M., 2016. Hurricane induced ocean waves and Stokes drift and their Impacts on surface transport and dispersion in the Gulf of Mexico. Geophys. Res. Lett., DOI:10.1002/2015GL067619.

d'Ovidio, F., Penna, A. D., Trull, T. W., Nencioli, F., Pujol, M.-I., Rio, M.-H., Park, W.-H., Cotte, C., Zhou, M., Blain, S., 2015. The biogeochemical structuring role of horizontal stirring: Lagrangian perspectives on iron delivery downstream of the kerguelen plateau. Biogeosciences 12, 5567-5581.

Ducet, N., Le Traon, P. Y., Reverdin, G., 2000. Global high-resolution mapping of ocean circulation from TOPEX/Poseidon and ERS-1 and -2. Journal of Geophysical Research 105 (C8), 19477.

Fox-Kemper, B., Ferrari, R., Hallberg, R., 2008. Parameterization of mixed-layer eddies. Part I: theory and diagnosis. J. Phys. Oceanogr. 38, 1145-1165.

Griffa, A., Piterbarg, L., Özgökmen, T. M., Jan 2004. Predictability of Lagrangian particle trajectories: Effects of smoothing of the underlying Eulerian flow. J. Mar. Res. 62 (1), $1-35$. 
Haller, G., Beron-Vera, F., 2013. Coherent Lagrangian vortices: The black holes of turbulence. Journal of Fluid Mechanics 731 (R4), 1-10.

Haller, G., Poje, A., 1998. Finite time transport in aperiodic flows. Physica D: Nonlinear Phenomena 119, 352-380.

Haller, G., Yuan, G., 2000. Lagrangian coherent structures and mixing in two-dimensional turbulence. Physica D 147, 352-370.

Haza, A., Özgökmen, T., Griffa, A., Garraffo, Z., Piterbarg, L., 2012. Parameterization of particle transport at submesoscales in the Gulf Stream region using Lagrangian subgridscale models. Ocean Modelling 42, 31-49.

Haza, A., Özgökmen, T., Griffa, A., Molcard, A., Poulain, P., Peggion, G., 2010. Transport properties in small scale coastal flows: relative dispersion from VHF radar measurements in the Gulf of La Spezia. Ocean Dynamics 60, 861-882.

Haza, A. C., Poje, A., Özgökmen, T. M., Martin, P., 2008. Relative dispersion from a highresolution coastal model of the Adriatic Sea. Ocean Modelling 22, 48-65.

Huntley, H., B.L. Lipphardt, J., Jacobs, G. A., A.D. Kirwan, J., 2015. Clusters, deformation, and dilation: Diagnostics for material accumulation regions. J. Geophys. Res. Oceans 120, $6622-6636$.

Jacobs, G. A., Huntley, H. S., A. D. Kirwan, J., B. L. Lipphardt, J., Campbell, T., Smith, T., Edwards, K., Bartels, B., 2015. Ocean processes underlying surface clustering. J. Geophys. Res. Oceans 121, 180-197.

Klein, P., Lapeyre, G., 2009. The oceanic vertical pump induced by mesoscale and submesoscale turbulence. Annu. Rev. Mar. Sci. 1, 351-375.

LaCasce, J. H., 2008. Statistics from Lagrangian observations. Prog. Ocean. 77/1, 1-29.

Large, W., McWilliams, J., Doney, S., 1994. Oceanic vertical mixing: A review and a model with a nonlocal boundary layer parameterization. Reviews of Geophysics 32 (4), 363-403.

Le Traon, P.-Y., Nadal, F., Ducet, N., 1998. An improved mapping method of multisatellite altimeter data. J. Atmos. Oceanic Technol. 15, 522-534.

Marshall, J., Shuckburgh, E., Jones, H., Hill, C., 2006. Estimates and implications of surface eddy diffusivity in the southern ocean derived from tracer transport. J. Phys. Oceanogr. 36, 1806-1821. 
McWilliams, J., 2008. Fluid dynamics at the margin of rotational control. Environ. Fluid Mech. 8, 441-449.

Mensa, J., Griffa, A., Garraffo, Z., Özgökmen, T., Haza, A., Veneziani, M., 2013. Seasonality of the submesoscale dynamics in the Gulf Stream region. Ocean Dynamics 63, 923-941.

Mezić, I., Wiggins, S., 1994. On the integrability and perturbation of three-dimensional fluid flows with symmetry. Journal of Nonlinear Science 4, 157-194.

Morey, S., Schroeder, W., O'Brien, J., Zavala-Hidalgo, J., 2003. The annual cycle of riverine influence in the eastern Mexico basin. Geophys. Res. Lett. 30, 1867.

Müller, P., McWilliams, J., Molemaker, M., 2005. Routes to dissipation in the ocean: the two-dimensional/three-dimensional turbulence conundrum. Marine Turbulence: Theories, Observations, and Models. Results of the CARTUM project, ed. H. Z. Baumert, J. Simpson and J. Sündermann. Cambridge University Press, 397-405.

Normile, D., 2014. Lost at sea. Science 344, 963-965.

Olascoaga, M., Haller, G., 2012. Forecasting sudden changes in environmental pollution patterns. Proc. Nat. Acad. Sci. 109, 4738-4743.

Olascoaga, M. J., Beron-Vera, F. J., Haller, G., Trinanes, J., Iskandarani, M., Coelho, E. F., Haus, B., Huntley, H. S., Jacobs, G., A. D. Kirwan, J., B.L. Lipphardt, J., Özgökmen, T. M., Reniers, A. J. H. M., Valle-Levinson, A., 2013. Drifter motion in the Gulf of Mexico constrained by altimetric Lagrangian coherent structures. Geophys. Res. Letters. 40, 6171-6175.

Olson, D., 1991. Rings in the ocean. Annu. Rev. Earth Planet Sci. 19, 293-311.

Özgökmen, T., Poje, A., Fischer, P., Childs, H., Krishnan, H., Garth, C., Haza, A., Ryan, E., 2012. On multi-scale dispersion under the influence of surface mixed layer instabilities and deep flows. Ocean Modelling 56, 16-30.

Özgökmen, T., Poje, A., Fischer, P., Haza, A., 2011. Large eddy simulations of mixed layer instabilities and sampling strategies. Ocean Modelling 39, 311-331.

Özgökmen, T. M., Fischer, P. F., 2012. CFD application to oceanic mixed layer sampling with Lagrangian platforms. International Journal of Computational Fluid Dynamics 26 (6-8), $1-12$.

Poje, A., Haza, A., Özgökmen, T., Magaldi, M., Garraffo, Z., 2010. Resolution dependent relative dispersion statistics in a hierarchy of ocean models. Ocean Modelling 31, 36-50. 
Poje, A. C., Özgökmen., T. M., Bruce Lipphart, J., Haus, B., Ryan, E. H., Haza, A. C., Jacobs, G., Reniers, A. J. H. M., Olascoaga, J., Novelli, G., Griffa, A., Beron-Vera, F. J., Chen, S., Hogan, P., Coelho, E., A. D. Kirwan, J., Huntley, H., Mariano, A. J., 2014. The nature of surface dispersion near the Deepwater Horizon oil spill. Proc. Natl. Acad. Sci. $111,12693-12698$.

Prasad, T., Hogan, P., 2007. Upper ocean response to hurricane ivan in a $1 / 25^{0}$ nested gulf of mexico hycom. J. Geophys. Res., 112:C04013.

Ramachandran, S., Tandon, A., Mahadevan, A., 2014. Enhancement in vertical fluxes at a front my mesoscale-submesoscale coupling. J. Geophys. Res. Oceans 119, 8495-8511.

Rio, M.-H., Hernandez, F., 2004. A mean dynamic topography computed over the world ocean from altimetry, in situ measurements, and a geoid model. J. Geophys. Res. 109, C12032.

Romero, I., Özgökmen, T. M., Snyder, S., Schwing, P., OMalley, B., Beron-Vera, F., Olascoaga, M., Zhu, P., Ryan, E., Chen, S., Wetzel, D., Hollander, D., Murawski, S., 2015. Tracking the Hercules 265 marine gas well blowout. J. Geophys. Res. Oceans 121, 706-724.

Rypina, I. I., Jayne, S., Yoshida, S., Macdonald, A., Buesseler, K., 2014. Drifter-based estimate of the 5 year dispersal of Fukushima-derived radionuclides. J. Geophys. Res. 119, 8177-8193.

Sagaut, P., 2006. Large eddy simulation for incompressible flows, 3rd Edition. Scientific Computation. Springer-Verlag, Berlin, an introduction, Translated from the 1998 French original, With forewords by Marcel Lesieur and Massimo Germano, With a foreword by Charles Meneveau.

Sanford, T., Kelly, K., Farmer, D., 2011. Sensing the ocean. Physics Today February, 24-28.

Sasaki, H., Klein, P., Qiu, B., Sasai, Y., 2014. Impact of oceanic-scale interactions on the seasonal modulation of ocean dynamics by the atmosphere. Nature Communications 5 , 5636.

Shcherbina, A. Y., D’Asaro, E. A., Lee, C. M., Klymak, J. M., Molemaker, M. J., McWilliams, J. C., 2013. Statistics of vertical vorticity, divergence, and strain in a developed submesoscale turbulence field. Geophysical Research Letters 40, 1-6.

Sturges, W., Lugo-Fernandez, A., Shargel, M., 2005. Introduction to circulation in the Gulf of Mexico. In 'Circulation in the Gulf of Mexico - Observations and Models" Eds: W. Sturges and Alexis Lugo-Fernandez, AGU monograph 161, 1-10. 
Taylor, J. R., Ferrari, R., 2010. Buoyancy and wind-driven convection at mixed layer density fronts. Journal of Physical Oceanography 40 (6), 1222-1242.

Thomas, L., Tandon, A., Mahadevan, A., 2008. Submesoscale processes and dynamics. Ocean modeling in an Eddying Regime, edited by M. Hecht and H. Hasume Geophysical Monograph $177,17-38$.

Walker, N. D., Jr, W. W., Jr., L. R., Babin, A., 2005. Effects of river discharge, wind stress, and slope eddies on circulation and satellite-observed structure of the Mississippi river plume. J. Coastal Res. 21, 1228-1244.

Wunsch, C., Stammer, D., 1998. Satellite altimetry, the marine geoid, and the oceanic general circulation. Ann. Rev. Earth Plan. Sci. 26, 219-253.

Zhong, Y., Bracco, A., Villareal, T., 2012. Pattern formation at the ocean surface: Sargassum distribution and the role of the eddy field. Limn. \& Ocean. Fluid and Environments 2, $12-27$. 


\section{List of Tables}

1 Flow direction discrepancies of a tracer launched on the edge of a Star Eddy barrier at day 15. The sampling is carried on along the attracting LCS of the filtered fields. The metrics used to quantify the differences between particles advected by the full flow and by the filtered flow are the averaged and std angle differences $(\Delta \theta)$ of the Lagrangian velocities, and the vector-rejection of the full flow velocities on the filtered velocities (corresponding to the velocity component perpendicular to the filtered velocities on the LCS of the filtered field) . . . . . . . . . . . . . . . . . . . . . .

\section{List of Figures}

1 (a) Surface salinity distribution in the Gulf of Mexico from the HYCOM simulation corresponding to Jan 15, 2010 (time=15 days). The Star Eddy is visible southeast of the Mississippi River delta. (b) Plan view of the Star Eddy and the surrounding flow on a different color scale. (c) Vertical salinity cross section. . . . . . . . . . . . . . . . . . . .

2 (a) Distribution of 1.2 million particles at time $=44$ days after they were launched uniformly over the Eddy at time=15 days. (b) Satellite (Meris) ocean color image of sargassum at $35^{\circ} \mathrm{N}, 61^{\circ} \mathrm{W}$. Note the striking similarity in the patterns between surface particle and sargassum distributions. (c) Spiral patterns in ocean eddy visualized by a massive green tide seen off the coast of Oman in February 13, 2013. . . . . . . . . . . . . . . . . . . .

3 Kinetic energy wavenumber spectrum of the HYCOM surface velocity field at day 35 (in black), low-passed by the Lanczos filter $G_{1}$ using $\lambda_{C}=20,40$, and $80 \mathrm{~km}$ (in blue). Superimposed is the kinetic energy spectrum of the geostrophic velocities obtained from the $G_{2}$-filtered SSH field, (in red). . . .

4 Surface speed $\left(\mathrm{m} \mathrm{s}^{-1}\right)$ in the modeled Gulf of Mexico, including the Loop Current and the Star Eddy for (a) original (unfiltered) HYCOM field, (b), (c), (d) $G_{1^{-}}$ filtered velocities with $\lambda_{C}=20,40$ and $80 \mathrm{~km}$, respectively. (e) Geostrophic velocities derived from $G_{2}$ filter. The geostrophic velocities on the shelf are not computed due to filtering errors and unreliability of SSH-anomalies in shallow waters. . . . .

5 Horizontal divergence, scaled by the local Coriolis parameter, in the vicinity of the Star Eddy, organized in the same way as in Fig. 4. . . . . . . . . . 
6 Scale-dependent FSLE $(\lambda(\delta))$ of the experiments described in Fig. 3. (a) $\lambda(\delta)$ computed from (a) original pairs, and (b) chance pairs. $\lambda(\delta)$ of AVISO is also included as a reference for satellite altimetry based results. The slopes mark the Richardson $(-2 / 3)$ and ballistic $(-1)$ regimes. . . . . . . . . . . . . . . . . 34

7 Particle concentrations on a log-scale 10 days after a square-launch centered on the Star Eddy, advected by the full flow (a), and the low-passed flows from the Lanczos filter with $\lambda_{C}=20 \mathrm{~km}(\mathrm{~b}), \lambda_{C}=40 \mathrm{~km}(\mathrm{c})$ and $\lambda_{C}=80 \mathrm{~km}(\mathrm{~d})$. Units are the number of particles per $0.001^{\circ} \times 0.001^{\circ}$ bin. . . . . . . . . . 35

8 Same as Fig. 7 after 31 days. . . . . . . . . . . . . . . . . . . . . . . 36

9 Same as Fig. 7 after 51 days. . . . . . . . . . . . . . . . . . 37

10 Attracting (backward FSLE in red) and repelling (forward FSLE in blue) LCSs in the vicinity of the Star Eddy at day=18 for the full field (a), and $G_{1}$-filtered fields with (b) $\lambda_{C}=20 \mathrm{~km}$, (c) $\lambda_{C}=40 \mathrm{~km}$, and (d) $\lambda_{C}=80$ $\mathrm{km}$. Units are in $\mathrm{hr}^{-1}$. Initial particle pair distance $\delta_{i} \approx 450 \mathrm{~m}$. Final pair distance $\delta_{f}=10 \times \delta_{i}$ for $\lambda_{c}=20$ and $40 \mathrm{~km}$, and a 10 day advection, and $\delta_{f}=20 \times \delta_{i}$ for $\lambda_{c}=80 \mathrm{~km}$, for a 30 day advection.

11 Evolution of a particle-based tracer launched on the inner edge of the SE (top left panel), as defined by the attracting LCS of the $G_{1}$ filtered field with $\lambda_{C}=80 \mathrm{~km}$. The advection of the tracer is done by the full field (in blue) and and by the filtered field (in red). The mesoscale attracting LCS ridges are displayed in the background in gray shades (darker shade for more attracting ridges $\ldots \ldots \ldots \ldots \ldots \ldots \ldots$

12 (a) Tracer distribution (in gray) at day $=55$ after being advected by the $G_{1}$-filtered (using $\lambda_{C}=80 \mathrm{~km}$ ) velocities along the edge of the Star Eddy eddy. Particles advected by the full flow and intersecting with the tracer have an averaged (over a $5 \mathrm{~km}$ radius) velocity represented by the blue vectors. The velocities of the filtered field at the same location are shown in red, and correspond to the flow evolving with the attracting LCS of the filtered field. The vector-rejections are shown in green. (b) Angle differences in flow directions between the full and filtered field along the attracting LCS occupied by the tracer.

13 Evolution of the tracer 15, 25, 35 and 45 days after launch when advected by the $G_{1}$-filtered ( $\lambda_{C}=80 \mathrm{~km}$ ) flow (in red) and the full flow (in blue and green), and superimposed on the attracting LCSs (in gray). The particles colored in green correspond to the portion of tracer within $5 \mathrm{~km}$ of an attracting LCS not presently occupied by the low-pass tracer counterpart. Any particle/tracer in this configuration has leaked through the mesoscale transport barriers, and is more likely to diverge from the original distribution at the fastest pace. . . . 
14 Time series of flow direction discrepancies averaged over the areas of the attracting LCS of $G_{1}$-filtered cases (occupied by the tracer) for (a) $\lambda_{C}=20 \mathrm{~km}$, (b) $\lambda_{C}=40 \mathrm{~km}$, and (c) $\lambda_{C}=80 \mathrm{~km}$, respectively. The shading areas are delimited by the standard deviations. . . . . . . . . . . . . . . . . . . . . . 42

15 Time series of percentages of the total number of particles having leaked to other attracting LCS unoccupied by the tracer for all filtered cases. . . . . .

16 Particle concentrations on a log-scale 11 (top), 31 (middle) and 51 (bottom) days after a square-launch centered on the Star Eddy, advected by the geostrophic velocities from the altimetry-proxy. . . . . . . . . . . . . . . . 44

17 Attracting LCS (backward in time FSLE) at day=35 computed from the full velocity field (top), the Lanczos filtered fields with $\lambda_{C}=80 \mathrm{~km}$ (middle), and the geostrophic velocities of the altimetry proxy (bottom). . . . . . . . . .

18 Same as Fig. 11 but the FSLE ridges and red particles are derived from field filtered by $G_{2}$, while the blue tracer shows the tracer advection by the full field. 46

19 (Left Panel) Tracer release location on year day $=173$ (June 22) in an eddy in the HYCOM GoM simulation. (Right Panel) Tracer distributions from the full (blue) and filtered (with $\lambda_{C}=80 \mathrm{~km}$ ) velocity fields (red) after 57 days (August 18) . . . . . . . . . . . . . . . . . . . . 47 


\begin{tabular}{|c|c|c|c|c|}
\hline Filter & $\begin{array}{l}\text { Day } \\
(2010)\end{array}$ & $\begin{array}{l}<\Delta \theta> \\
\text { Radius }=5 \mathrm{~km}\end{array}$ & $\operatorname{std}(\Delta \theta)$ & $\begin{array}{l}\text { Vector rejection } \\
\% \text { of Lag. }\|\mathbf{V}\|)\end{array}$ \\
\hline \multicolumn{5}{|l|}{$\overline{G_{1}}$} \\
\hline \multirow[t]{4}{*}{$\lambda_{C}=20 \mathrm{~km}$} & 25 & $-2^{o}$ & $14^{\circ}$ & $4 \%$ \\
\hline & 35 & $15^{\circ}$ & $15^{\circ}$ & $9 \%$ \\
\hline & 45 & $-2^{o}$ & $23^{\circ}$ & $3 \%$ \\
\hline & 55 & $-5^{o}$ & $37^{\circ}$ & $9 \%$ \\
\hline \multirow[t]{4}{*}{$\lambda_{C}=40 \mathrm{~km}$} & 25 & $-3^{o}$ & $15^{\circ}$ & $4 \%$ \\
\hline & 35 & $14^{o}$ & $18^{\circ}$ & $5 \%$ \\
\hline & 45 & $1^{o}$ & $24^{\circ}$ & $2 \%$ \\
\hline & 55 & $-6^{o}$ & $27^{\circ}$ & $6 \%$ \\
\hline \multirow[t]{4}{*}{$\lambda_{C}=80 \mathrm{~km}$} & 25 & $1^{o}$ & $12^{o}$ & $4 \%$ \\
\hline & 35 & $7^{\circ}$ & $22^{\circ}$ & $4 \%$ \\
\hline & 45 & $-6^{o}$ & $29^{\circ}$ & $3 \%$ \\
\hline & 55 & $4^{o}$ & $51^{\circ}$ & $7 \%$ \\
\hline \multicolumn{5}{|l|}{$G_{2}$} \\
\hline & 25 & $-10^{\circ}$ & $18^{\circ}$ & $11 \%$ \\
\hline & 35 & $12^{o}$ & $16^{o}$ & $10 \%$ \\
\hline & 45 & $4^{o}$ & $20^{\circ}$ & $8 \%$ \\
\hline & 55 & $-6^{o}$ & $16^{o}$ & $5 \%$ \\
\hline
\end{tabular}

Table 1: Flow direction discrepancies of a tracer launched on the edge of a Star Eddy barrier at day 15. The sampling is carried on along the attracting LCS of the filtered fields. The metrics used to quantify the differences between particles advected by the full flow and by the filtered flow are the averaged and std angle differences $(\Delta \theta)$ of the Lagrangian velocities, and the vector-rejection of the full flow velocities on the filtered velocities (corresponding to the velocity component perpendicular to the filtered velocities on the LCS of the filtered field). 
(a)

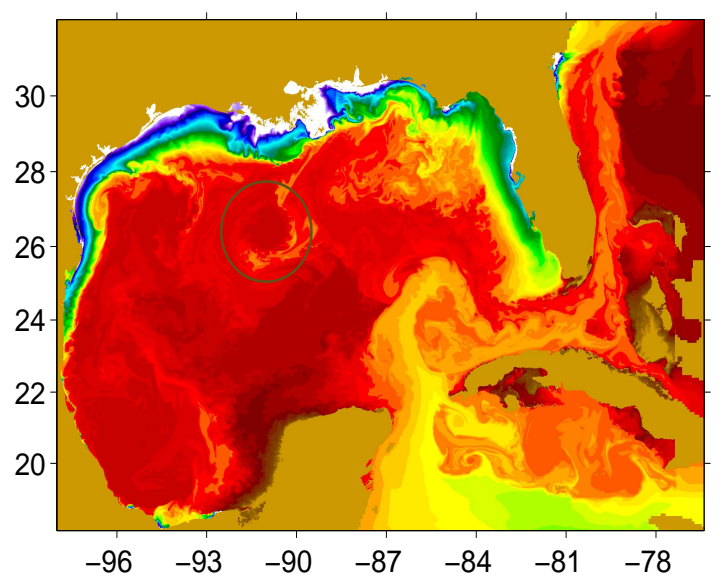

(b)

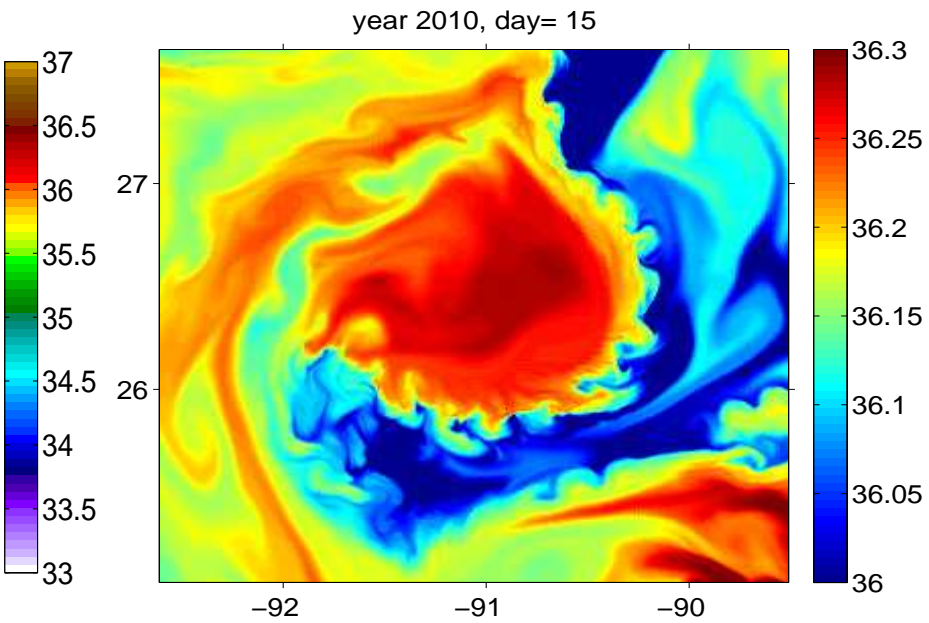

(c)

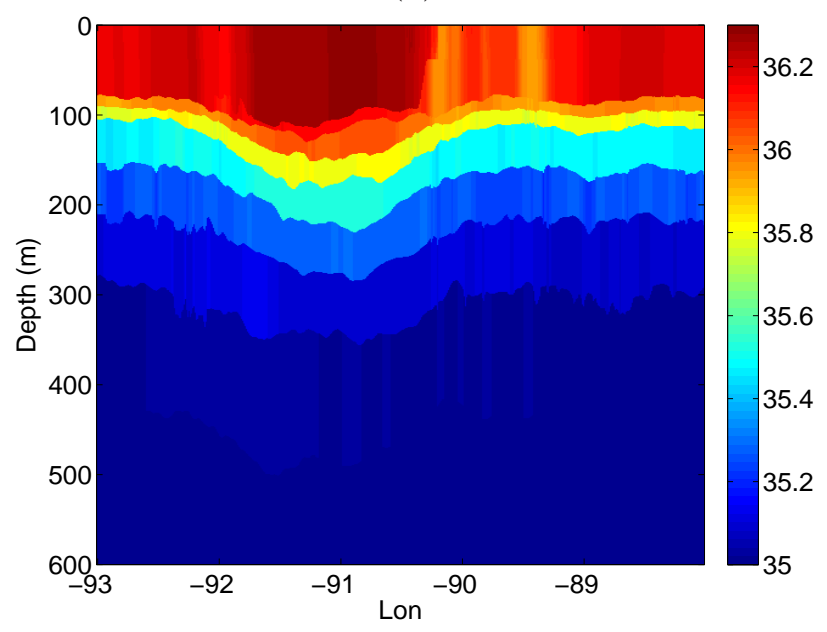

Figure 1: (a) Surface salinity distribution in the Gulf of Mexico from the HYCOM simulation corresponding to Jan 15, 2010 (time=15 days). The Star Eddy is visible southeast of the Mississippi River delta. (b) Plan view of the Star Eddy and the surrounding flow on a different color scale. (c) Vertical salinity cross section. 
(a)

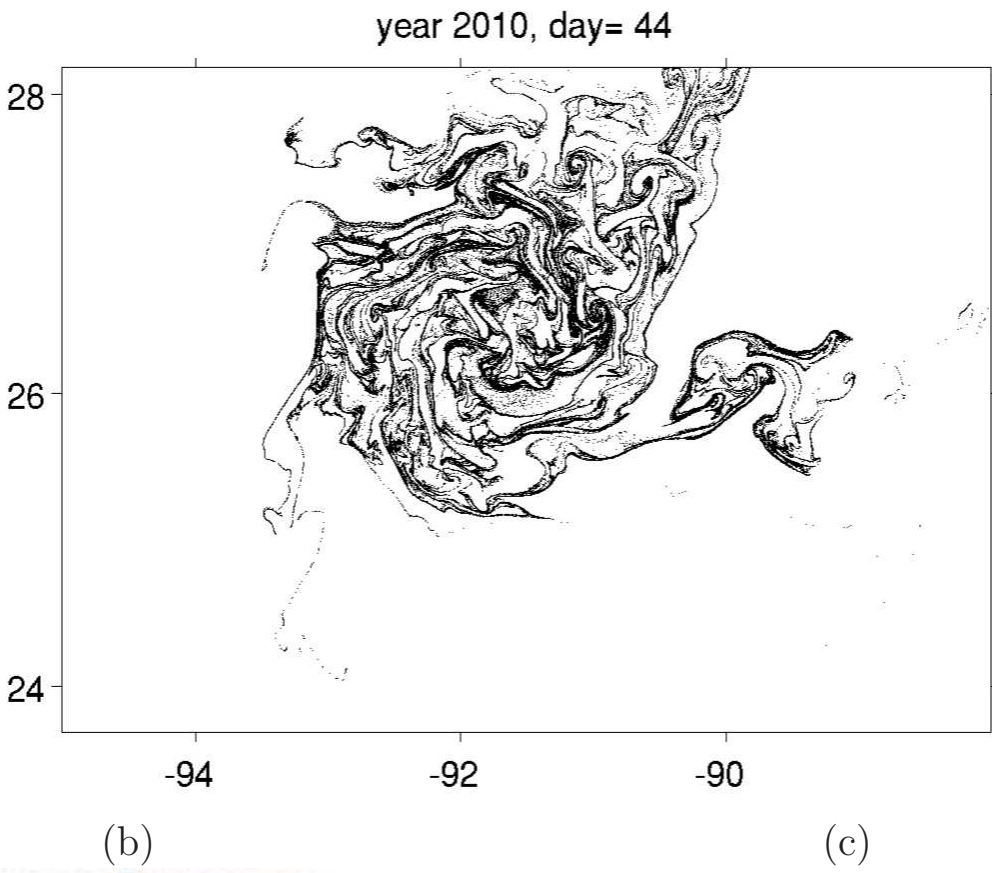

MERIS 2008 image of saraassum at $35 \mathrm{~N}, 61 \mathrm{~W}, 45 \mathrm{~km}$ across
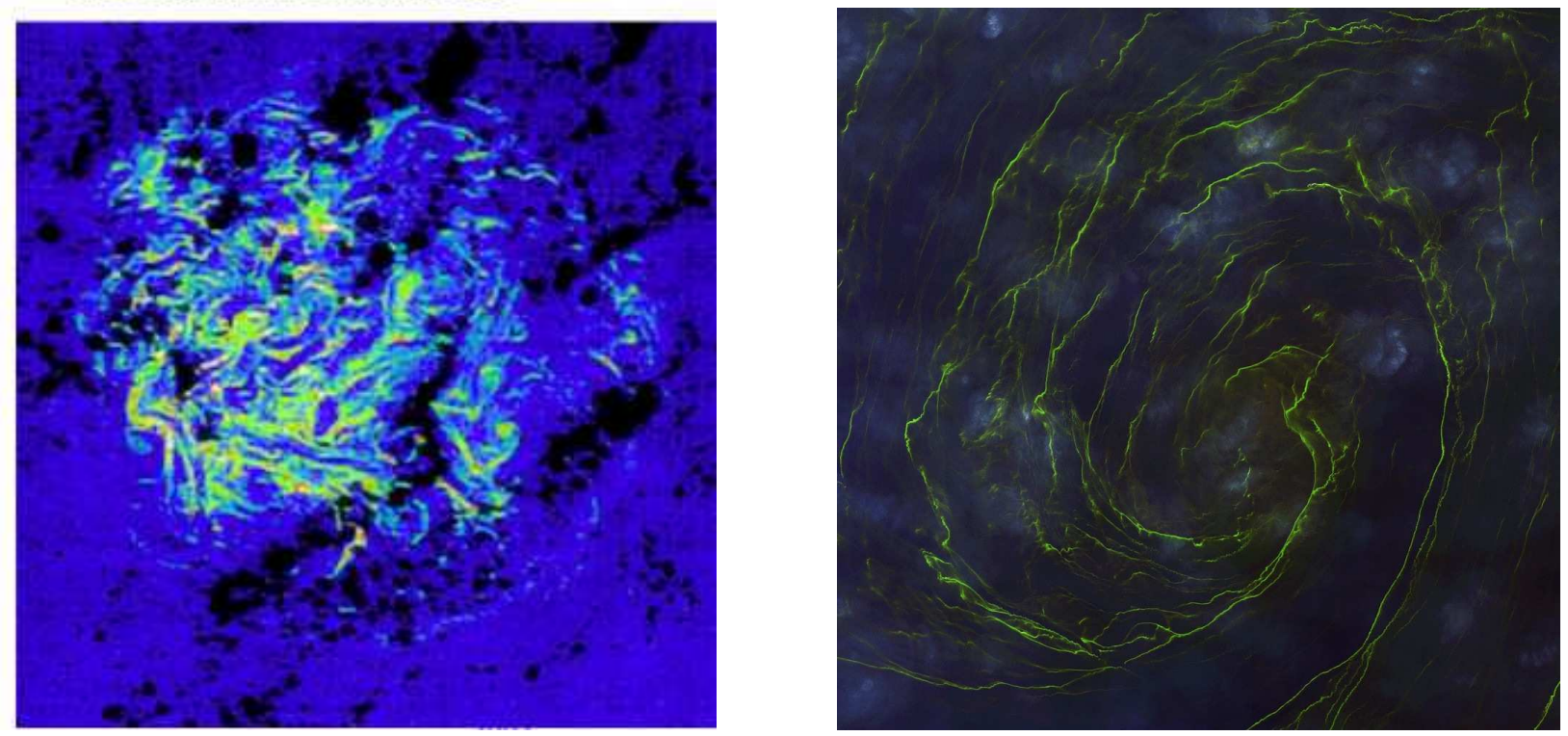

Figure 2: (a) Distribution of 1.2 million particles at time $=44$ days after they were launched uniformly over the Eddy at time=15 days. (b) Satellite (Meris) ocean color image of sargassum at $35^{\circ} \mathrm{N}, 61^{\circ} \mathrm{W}$. Note the striking similarity in the patterns between surface particle and sargassum distributions. (c) Spiral patterns in ocean eddy visualized by a massive green tide seen off the coast of Oman in February 13, 2013. 


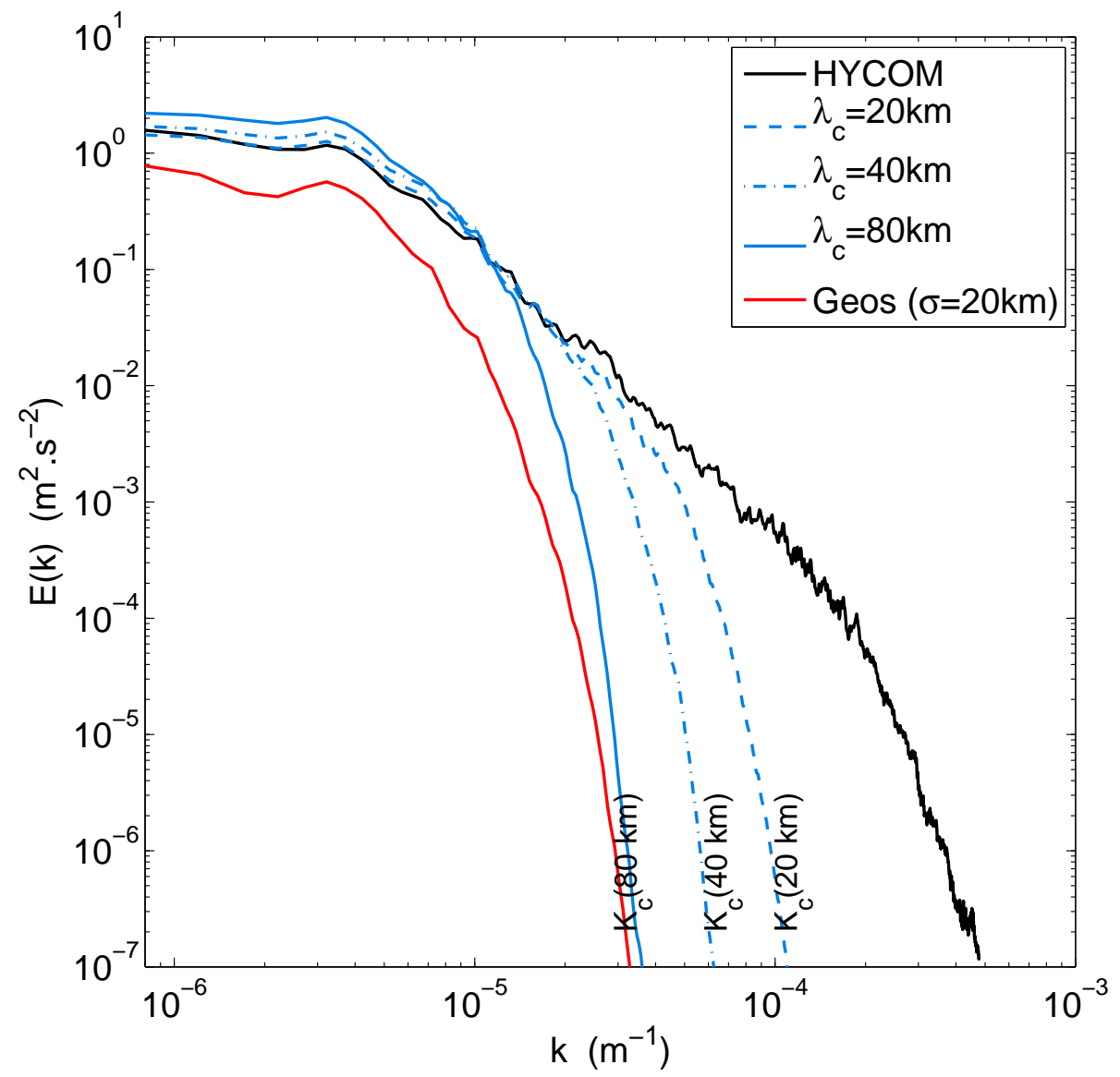

Figure 3: Kinetic energy wavenumber spectrum of the HYCOM surface velocity field at day 35 (in black), low-passed by the Lanczos filter $G_{1}$ using $\lambda_{C}=20,40$, and $80 \mathrm{~km}$ (in blue). Superimposed is the kinetic energy spectrum of the geostrophic velocities obtained from the $G_{2}$-filtered SSH field, (in red). 
(a)

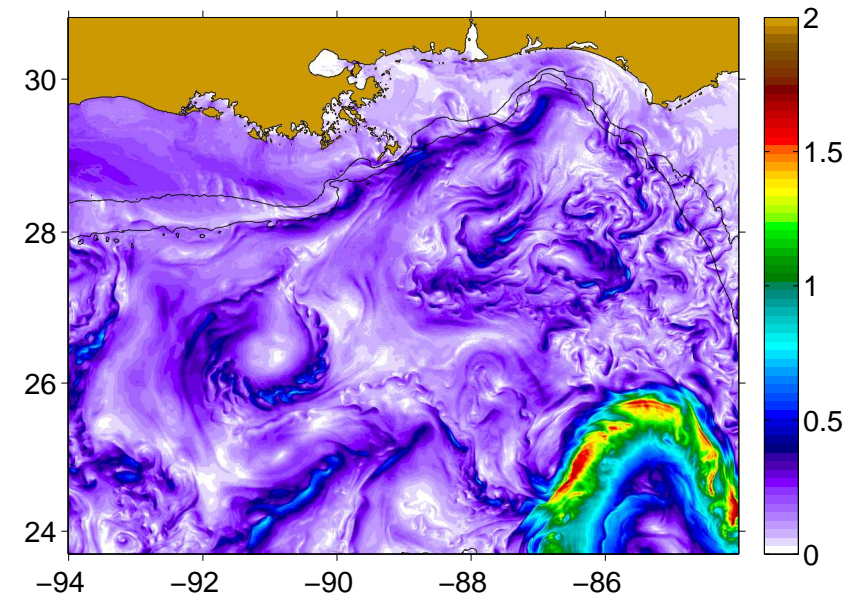

(b)

(c)

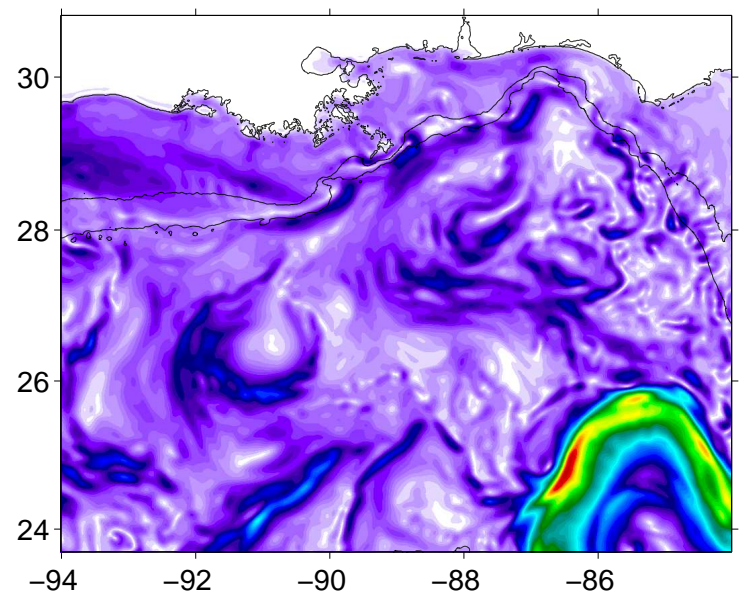

(d)

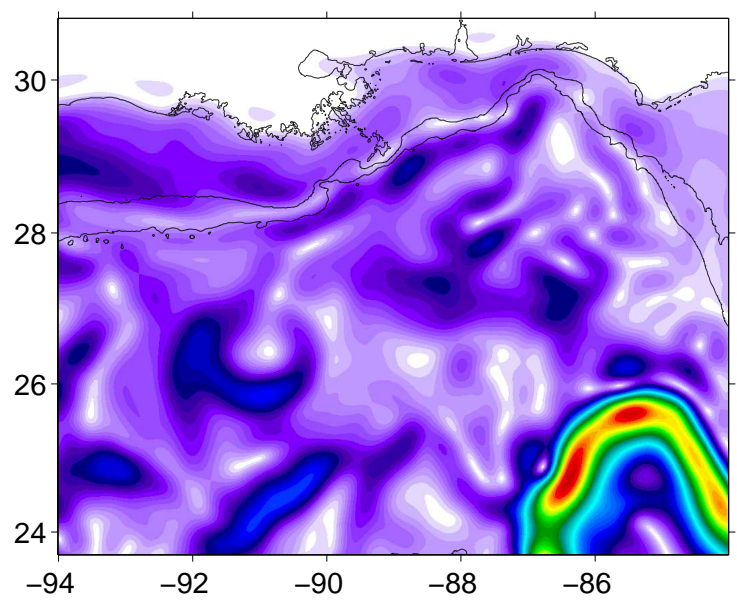

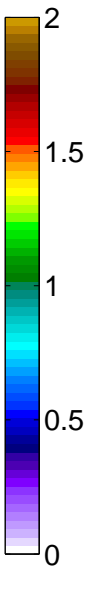

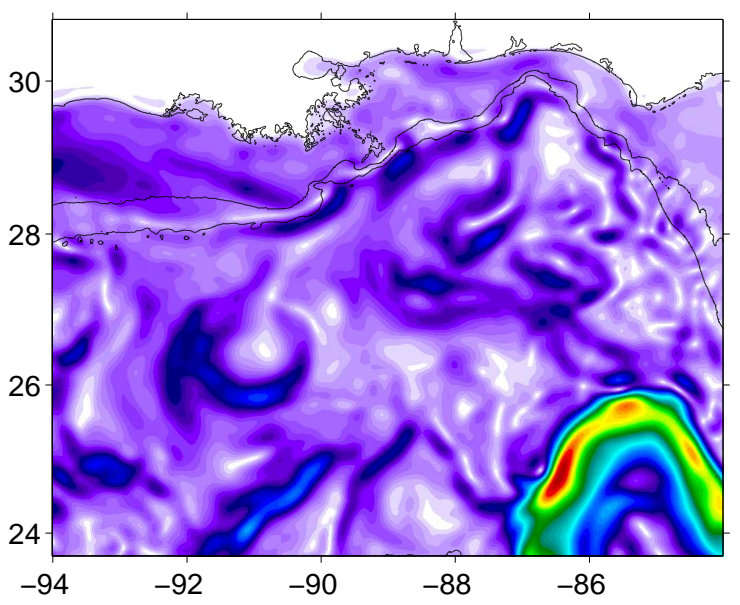

(e)

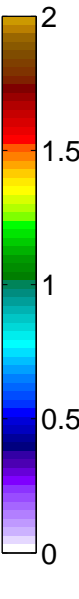

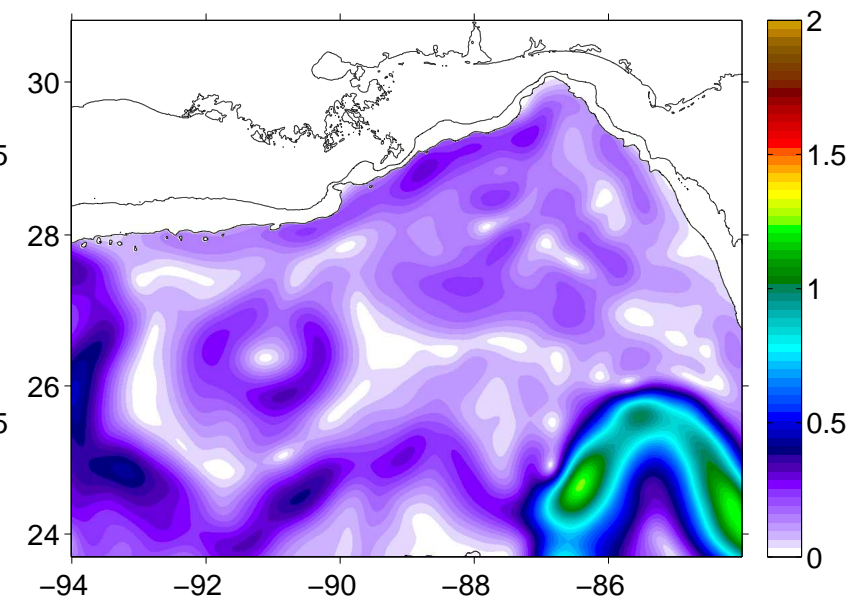

Figure 4: Surface speed $\left(\mathrm{m} \mathrm{s}^{-1}\right)$ in the modeled Gulf of Mexico, including the Loop Current and the Star Eddy for (a) original (unfiltered) HYCOM field, (b), (c), (d) $G_{1}$-filtered velocities with $\lambda_{C}=20,40$ and $80 \mathrm{~km}$, respectively. (e) Geostrophic velocities derived from $G_{2}$ filter. The geostrophic velocities on the shelf are not computed due to filtering errors and unreliability of $\mathrm{SSH}$-anomalies in shallow waters. 
(a)

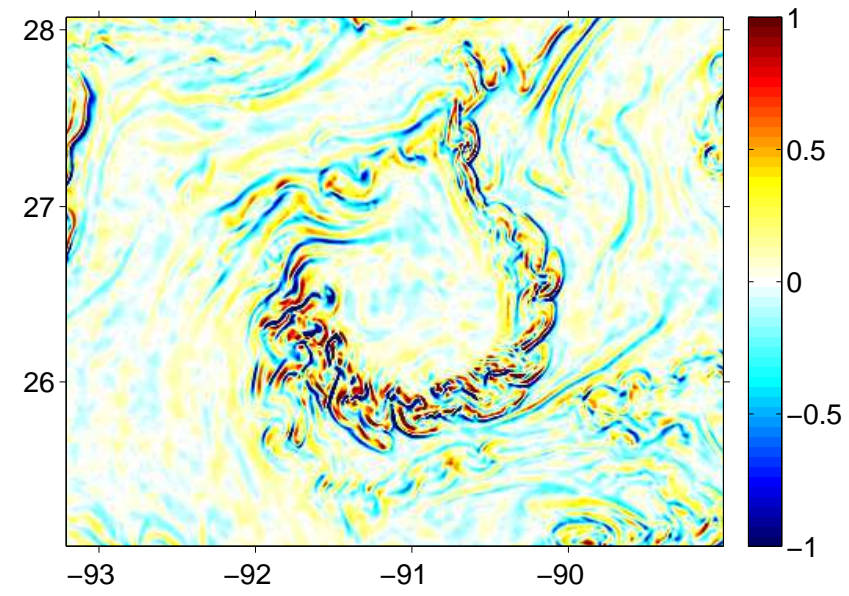

(b)

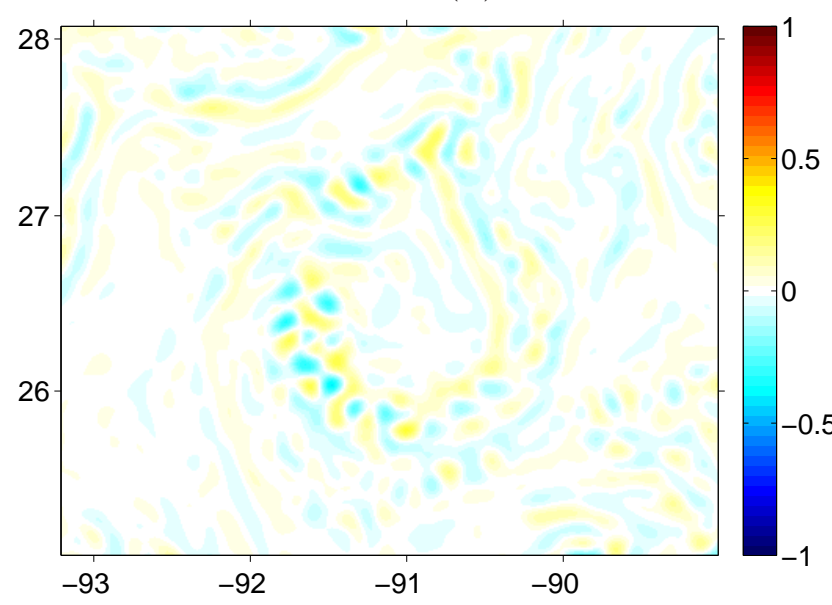

(d)

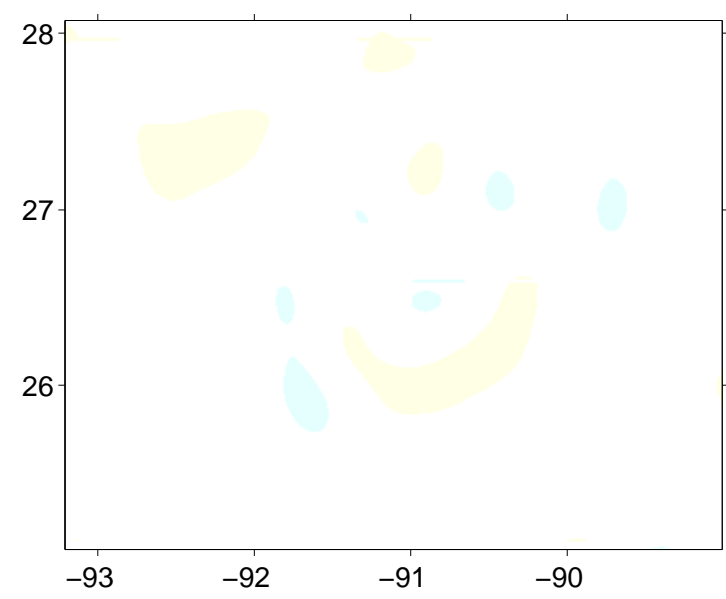

(c)

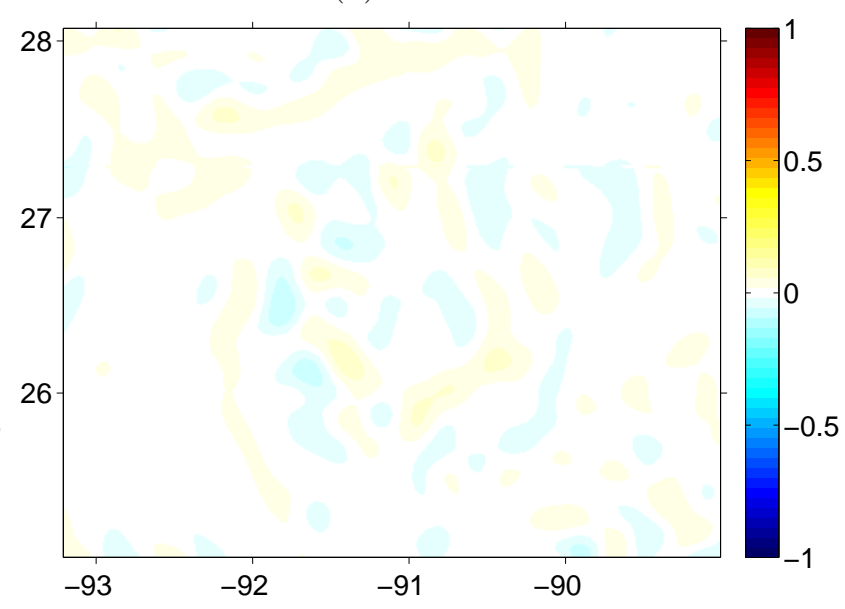

(e)

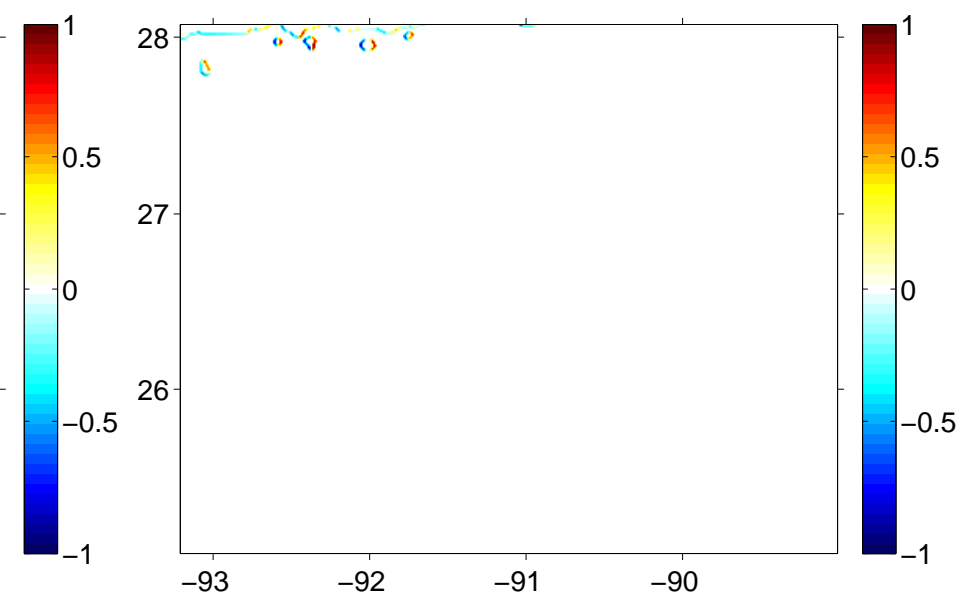

Figure 5: Horizontal divergence, scaled by the local Coriolis parameter, in the vicinity of the Star Eddy, organized in the same way as in Fig. 4. 
(a)

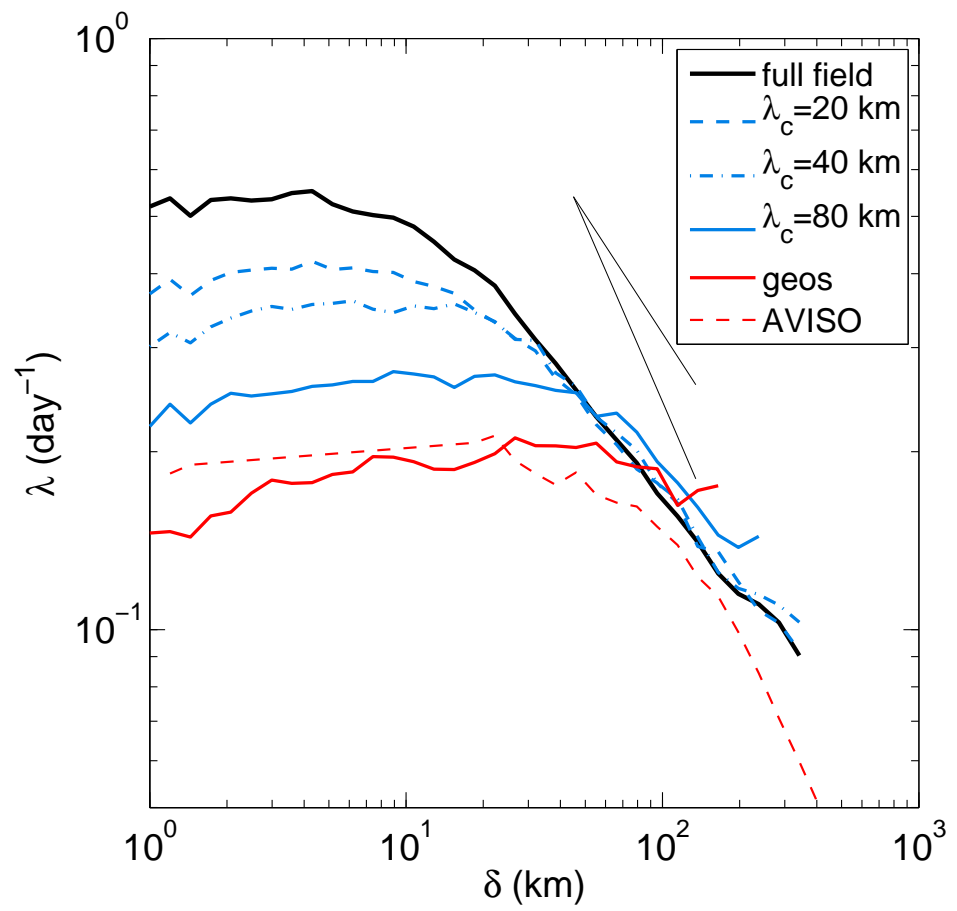

(b)

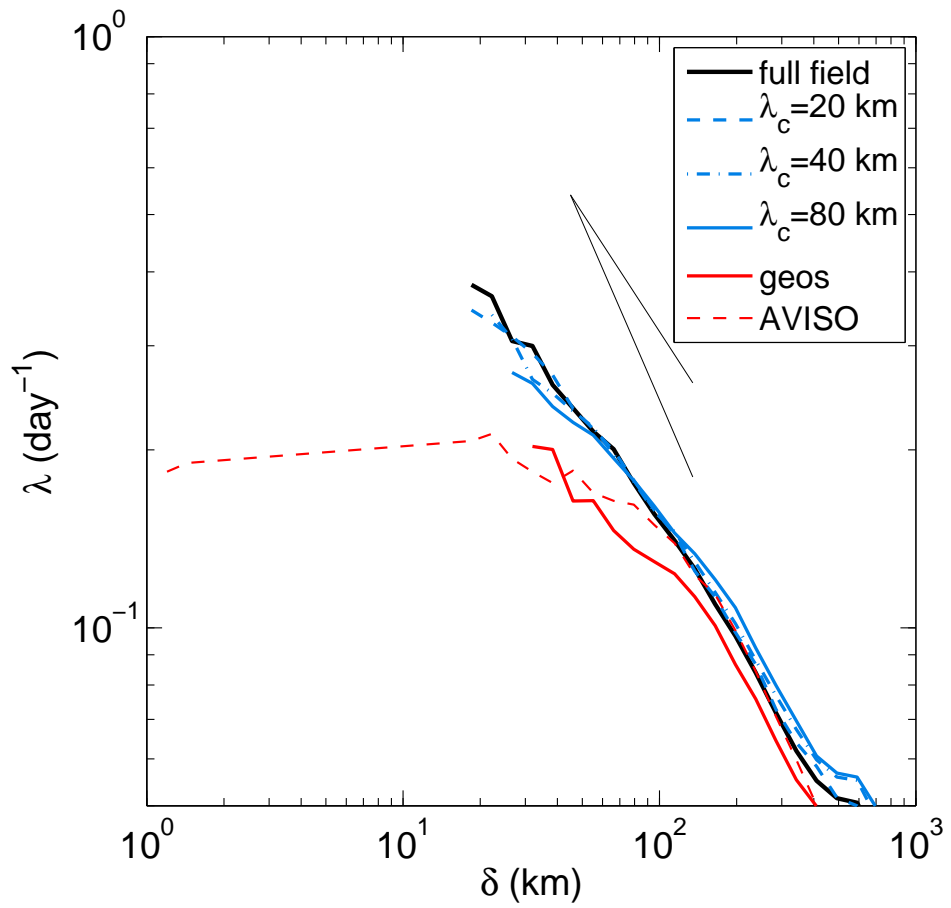

Figure 6: Scale-dependent FSLE $(\lambda(\delta))$ of the experiments described in Fig. 3. (a) $\lambda(\delta)$ computed from (a) original pairs, and (b) chance pairs. $\lambda(\delta)$ of AVISO is also included as a reference for satellite altimetry based results. The slopes mark the Richardson $(-2 / 3)$ and ballistic (-1) regimes. 
(a)

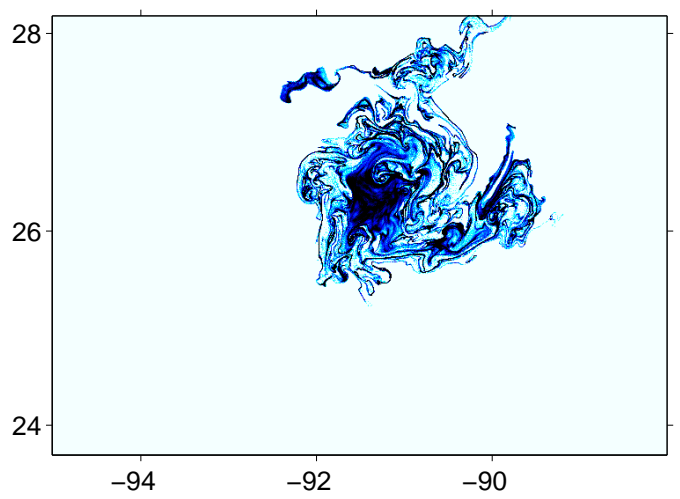

(c)

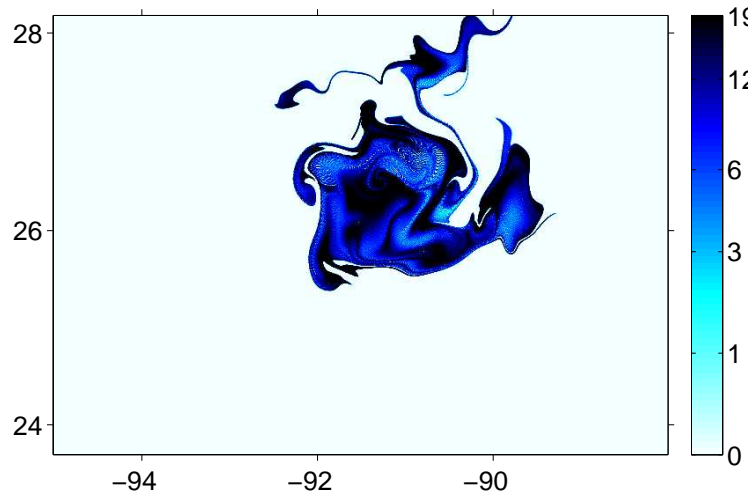

(b)
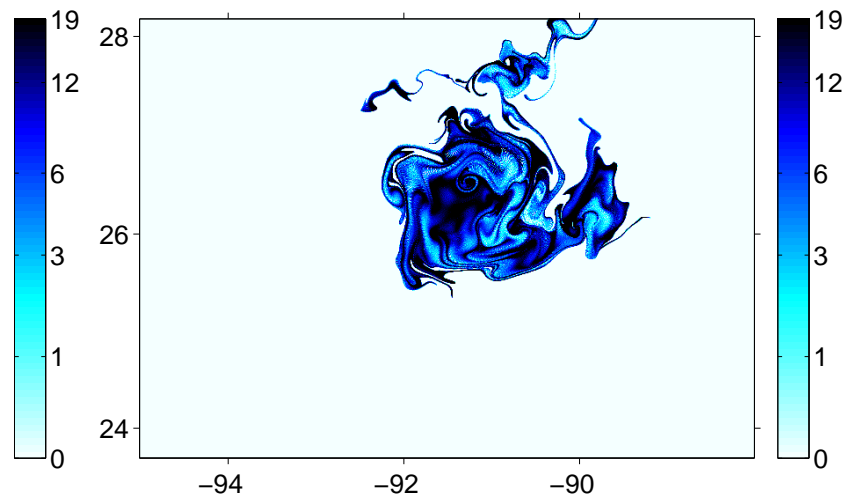

(d)

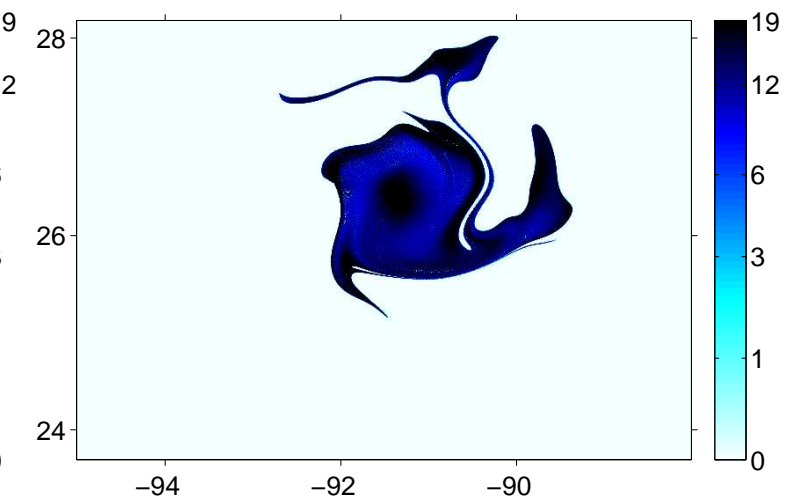

Figure 7: Particle concentrations on a log-scale 10 days after a square-launch centered on the Star Eddy, advected by the full flow (a), and the low-passed flows from the Lanczos filter with $\lambda_{C}=20 \mathrm{~km}(\mathrm{~b}), \lambda_{C}=40 \mathrm{~km}$ (c) and $\lambda_{C}=80 \mathrm{~km}$ (d). Units are the number of particles per $0.001^{\circ} \times 0.001^{\circ}$ bin. 
(a)

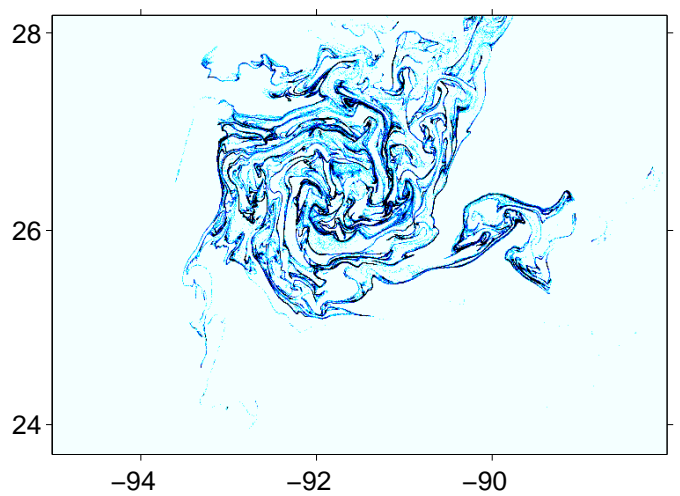

(c)

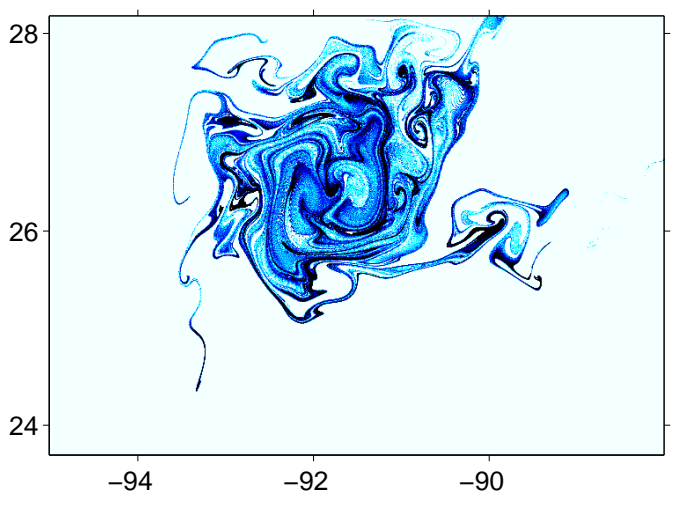

(b)
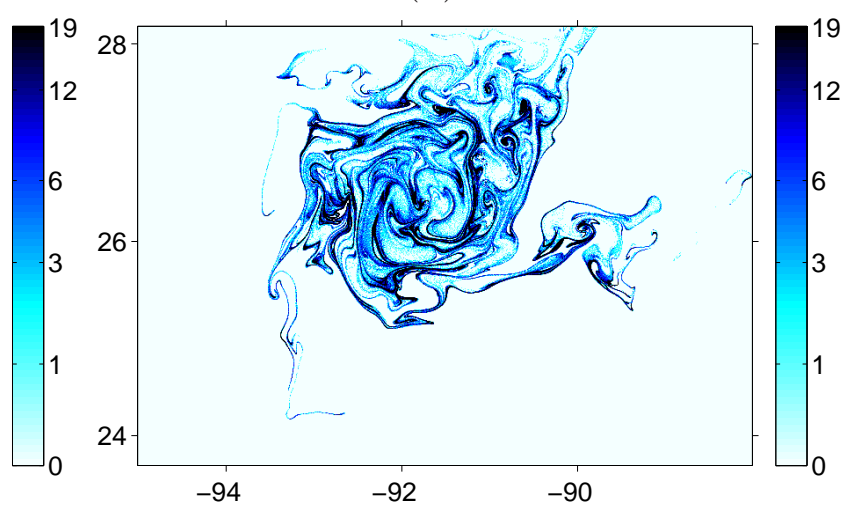

(d)

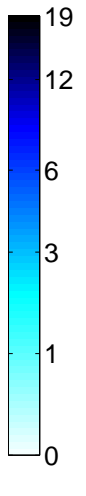

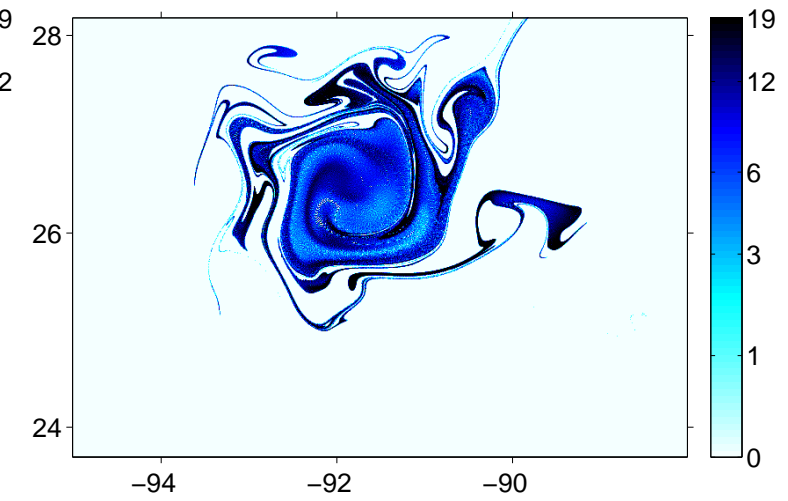

Figure 8: Same as Fig. 7 after 31 days. 
(a)

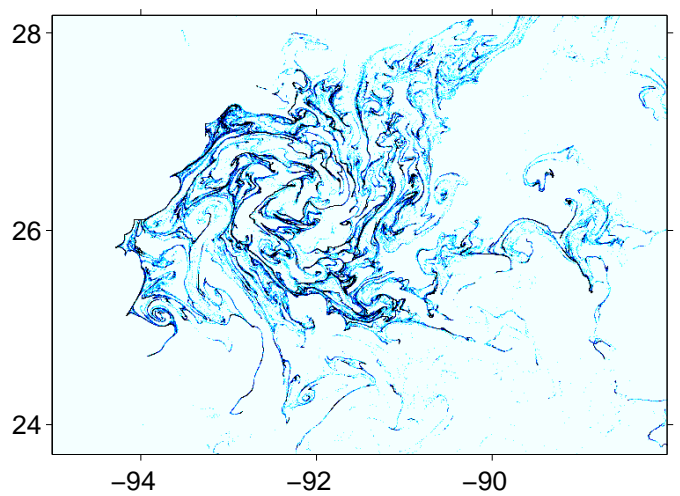

(c)

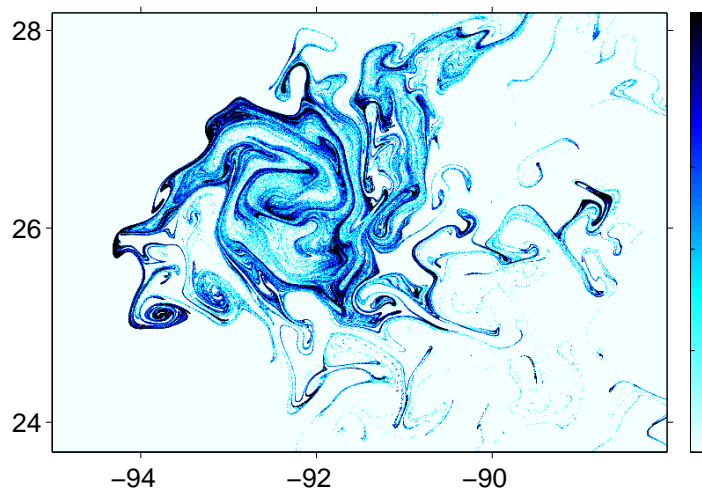

(b)
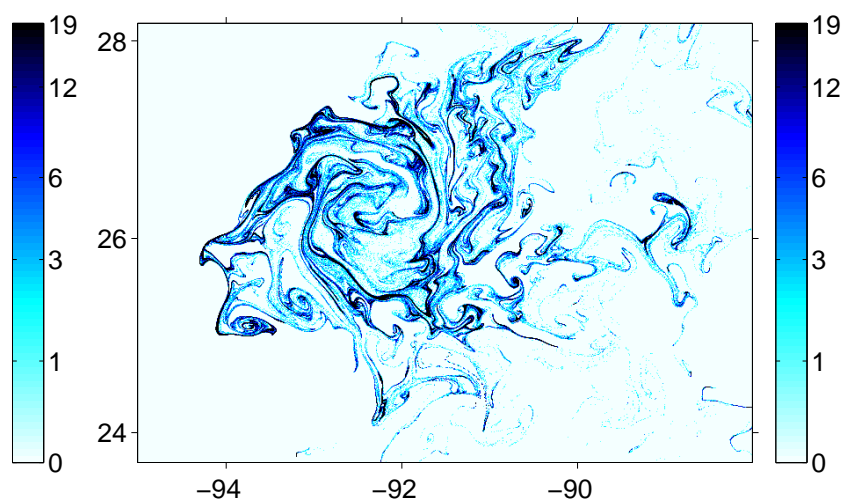

(d)

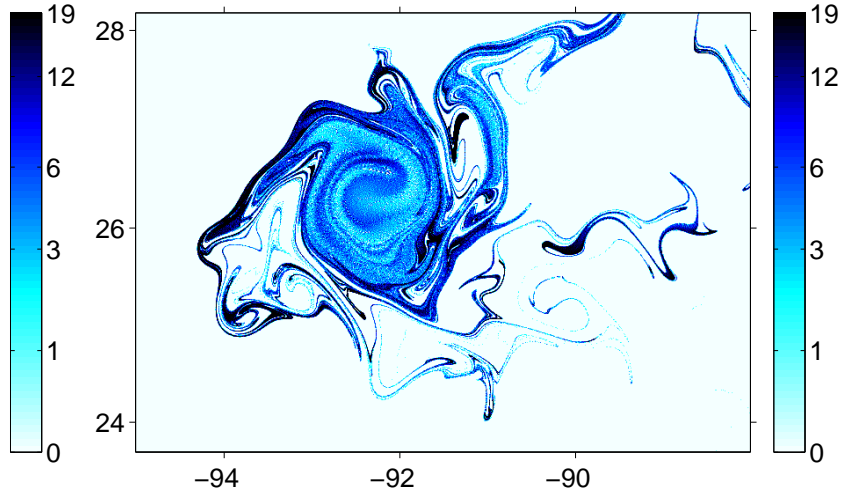

Figure 9: Same as Fig. 7 after 51 days. 
(a)

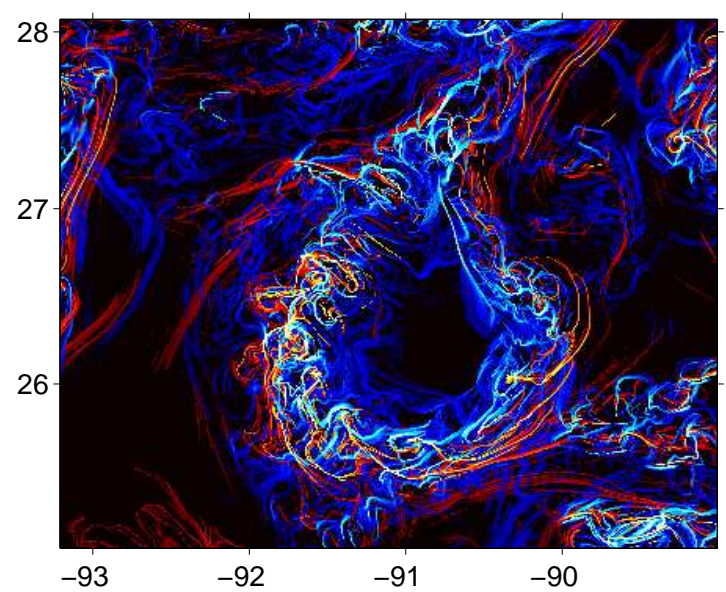

(c)

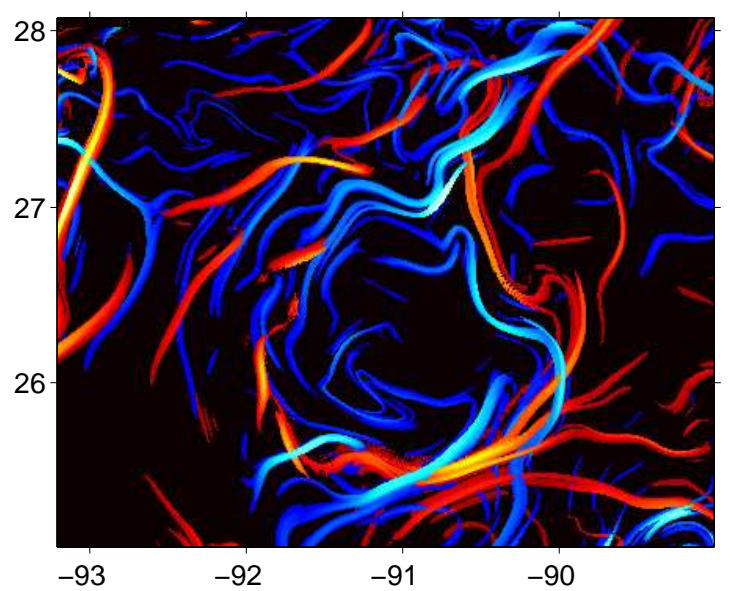

(b)
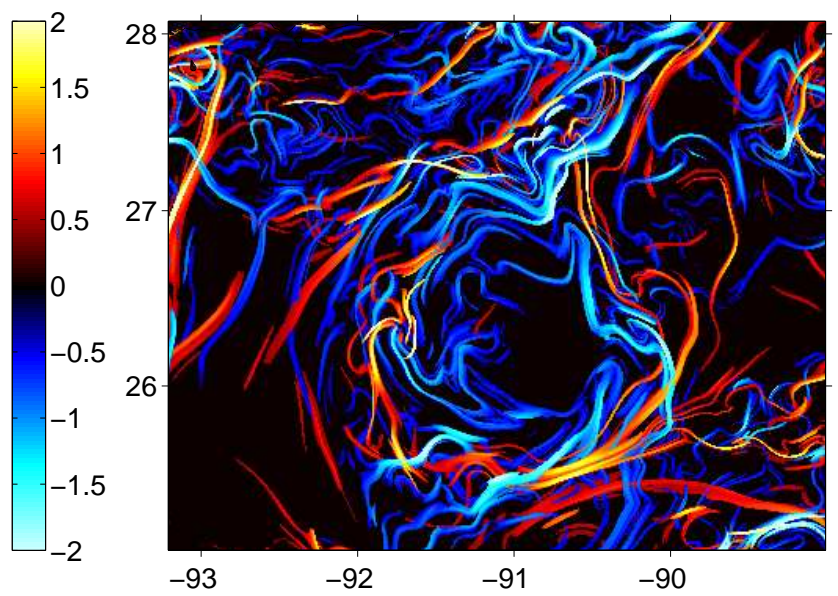

(d)

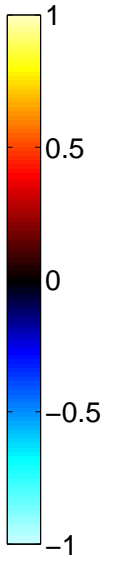

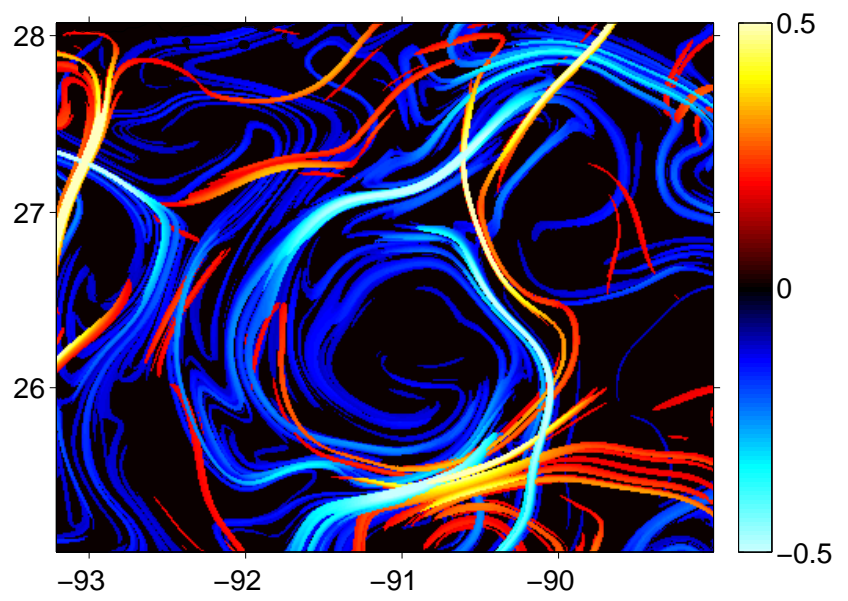

Figure 10: Attracting (backward FSLE in red) and repelling (forward FSLE in blue) LCSs in the vicinity of the Star Eddy at day=18 for the full field (a), and $G_{1}$-filtered fields with (b) $\lambda_{C}=20 \mathrm{~km},(\mathrm{c}) \lambda_{C}=40 \mathrm{~km}$, and (d) $\lambda_{C}=80 \mathrm{~km}$. Units are in $\mathrm{hr}^{-1}$. Initial particle pair distance $\delta_{i} \approx 450 \mathrm{~m}$. Final pair distance $\delta_{f}=10 \times \delta_{i}$ for $\lambda_{c}=20$ and $40 \mathrm{~km}$, and a 10 day advection, and $\delta_{f}=20 \times \delta_{i}$ for $\lambda_{c}=80 \mathrm{~km}$, for a 30 day advection. 

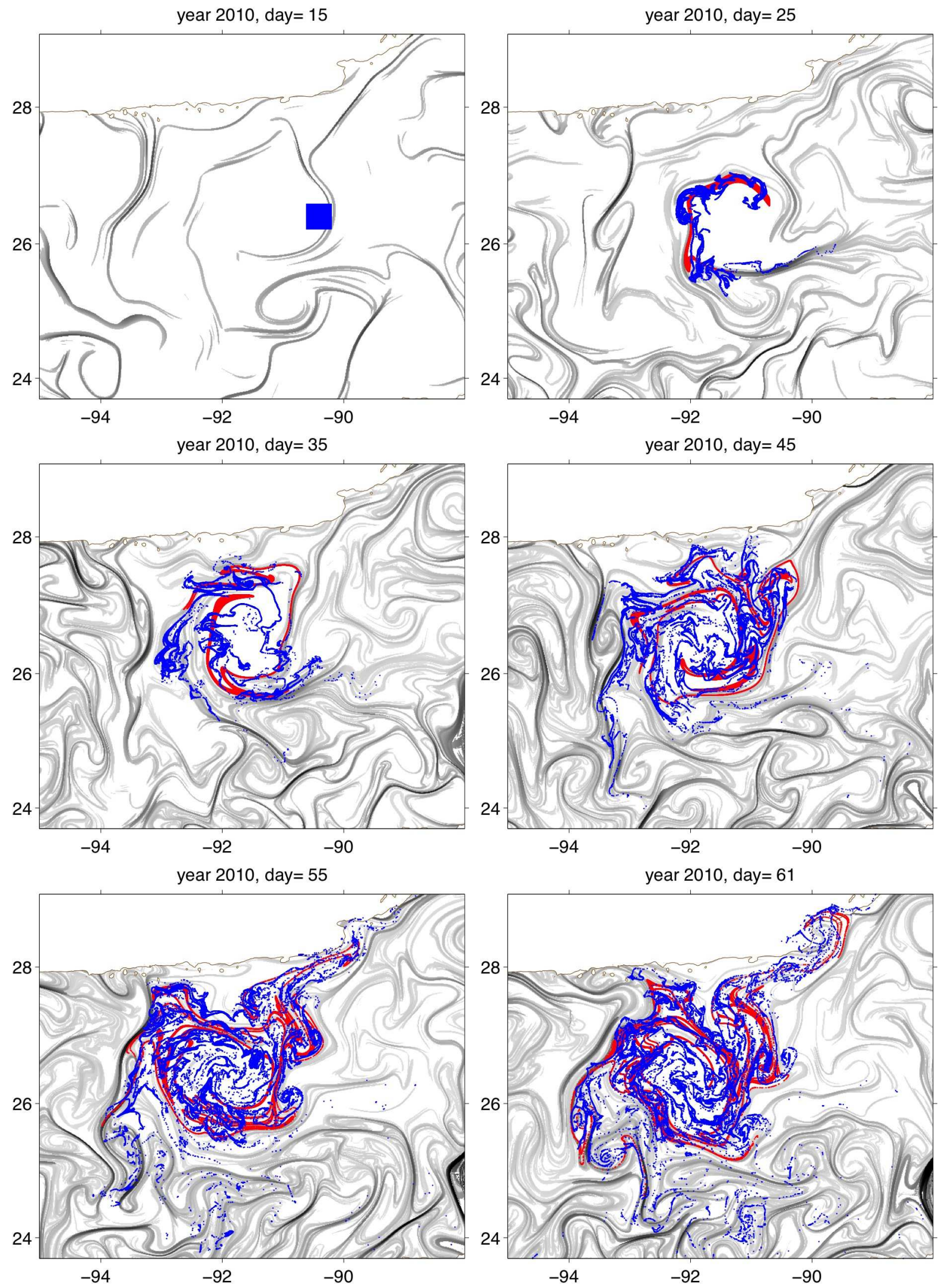

Figure 11: Evolution of a particle-based tracer launched on the inner edge of the SE (top left panel), as defined by the attracting LCS of the $G_{1}$ filtered field with $\lambda_{C}=80 \mathrm{~km}$. The advection of the tracer is done by the full field (in blue) and and by the filtered field (in red). The mesoscale attracting LCS ridges are displayed in the background in gray shades (darker shade for more attracting ridges) 
(a)

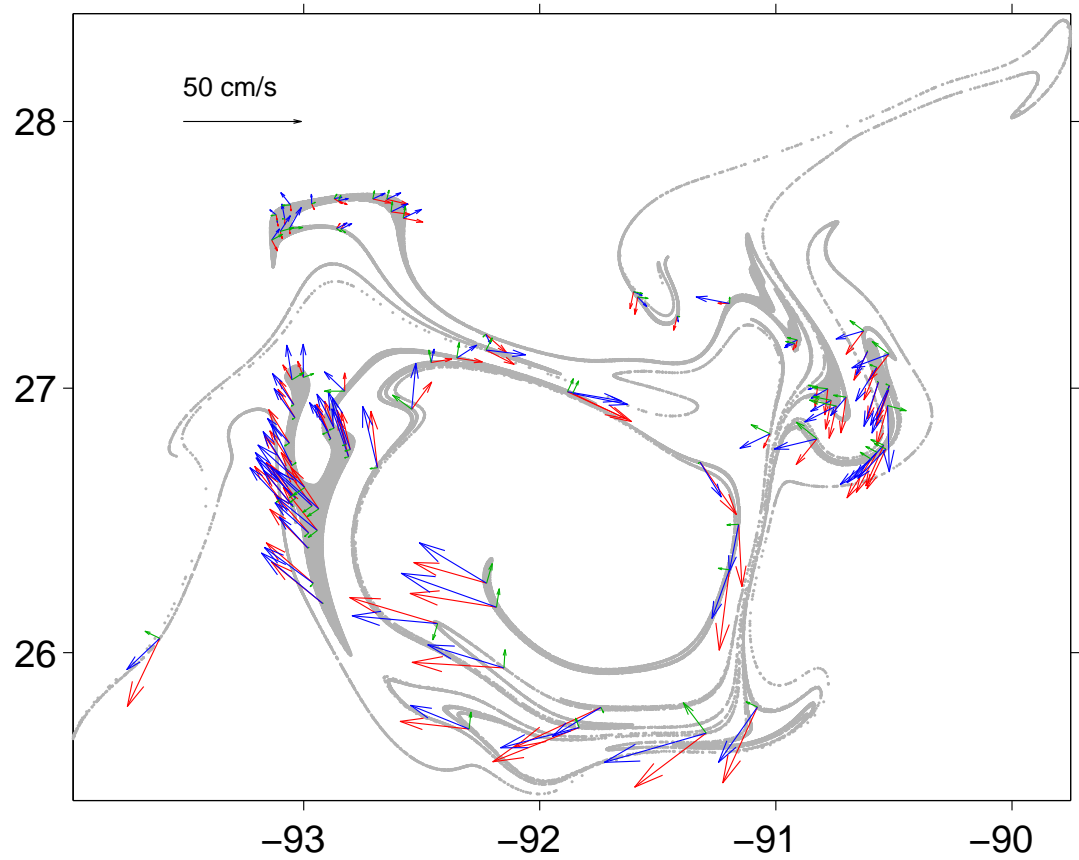

(b)

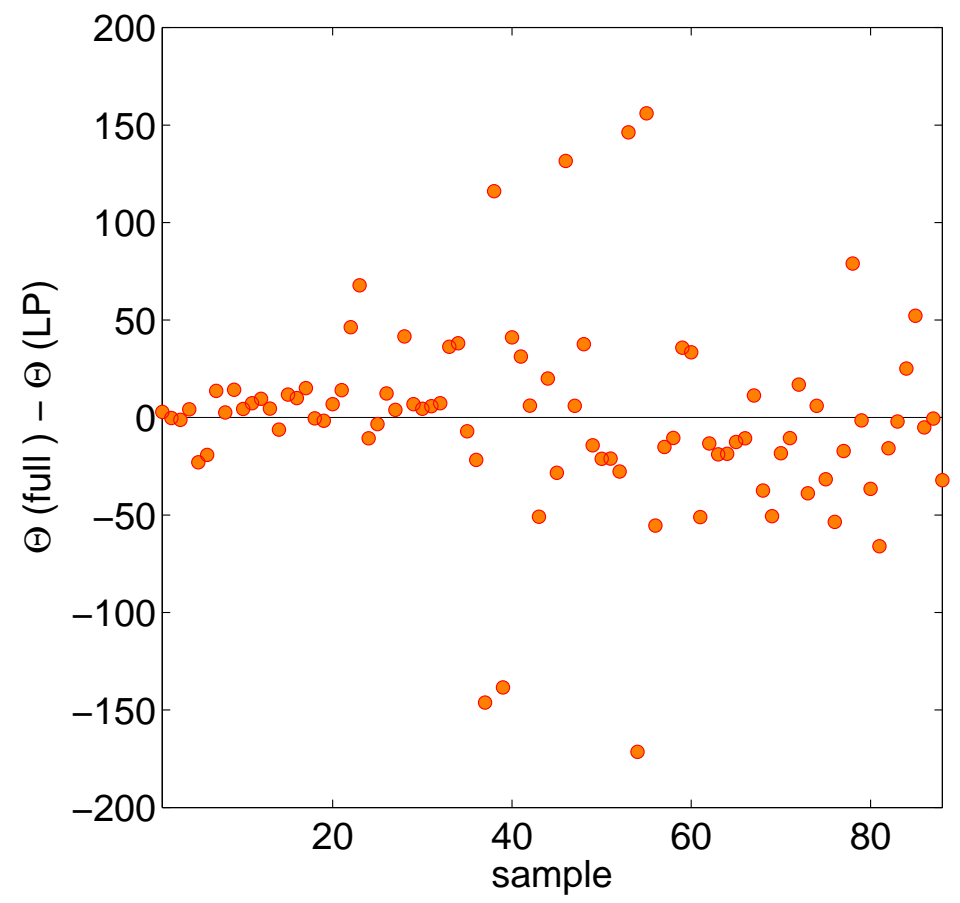

Figure 12: (a) Tracer distribution (in gray) at day=55 after being advected by the $G_{1}$-filtered (using $\lambda_{C}=80 \mathrm{~km}$ ) velocities along the edge of the Star Eddy eddy. Particles advected by the full flow and intersecting with the tracer have an averaged (over a $5 \mathrm{~km}$ radius) velocity represented by the blue vectors. The velocities of the filtered field at the same location are shown in red, and correspond to the flow evolving with the attracting LCS of the filtered field. The vector-rejections are shown in green. (b) Angle differences in flow directions between the full and filtered field along the attracting LCS occupied by the tracer. 

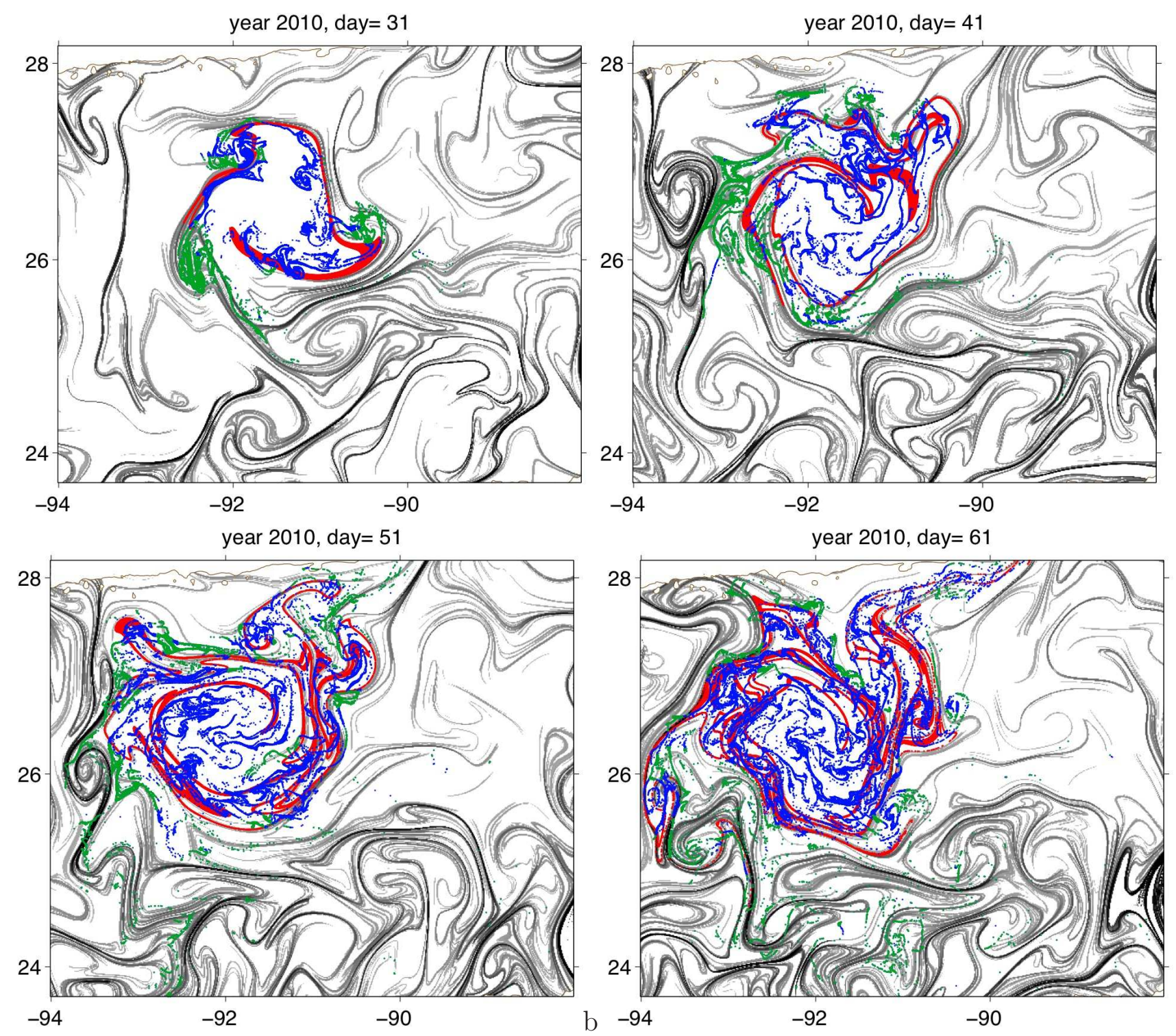

Figure 13: Evolution of the tracer 15, 25, 35 and 45 days after launch when advected by the $G_{1}$-filtered ( $\lambda_{C}=80 \mathrm{~km}$ ) flow (in red) and the full flow (in blue and green), and superimposed on the attracting LCSs (in gray). The particles colored in green correspond to the portion of tracer within $5 \mathrm{~km}$ of an attracting LCS not presently occupied by the low-pass tracer counterpart. Any particle/tracer in this configuration has leaked through the mesoscale transport barriers, and is more likely to diverge from the original distribution at the fastest pace. 


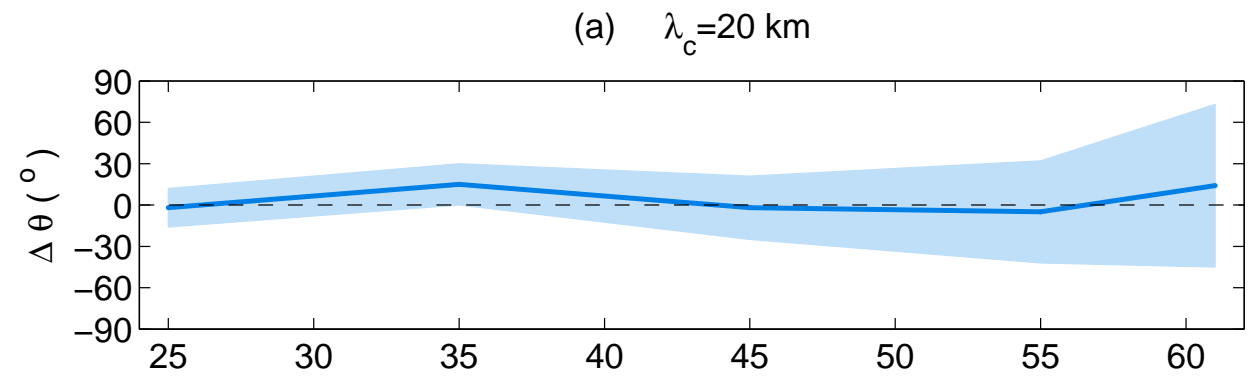

(b) $\lambda_{\mathrm{c}}=40 \mathrm{~km}$

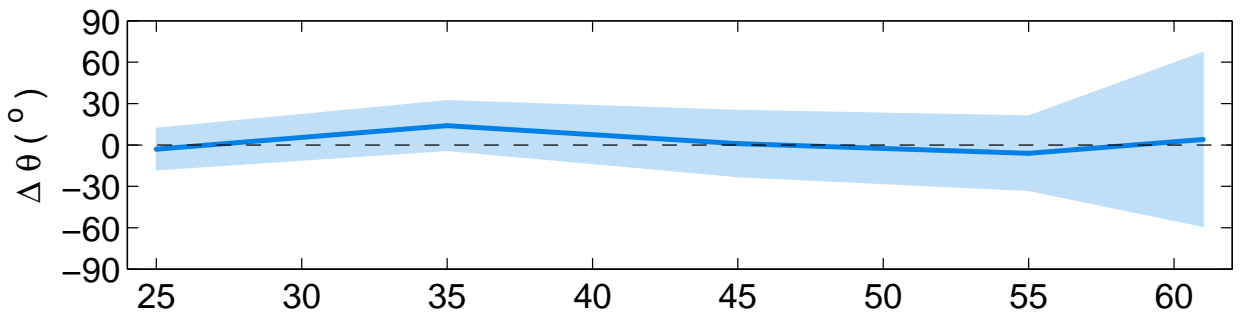

(c) $\lambda_{\mathrm{c}}=80 \mathrm{~km}$

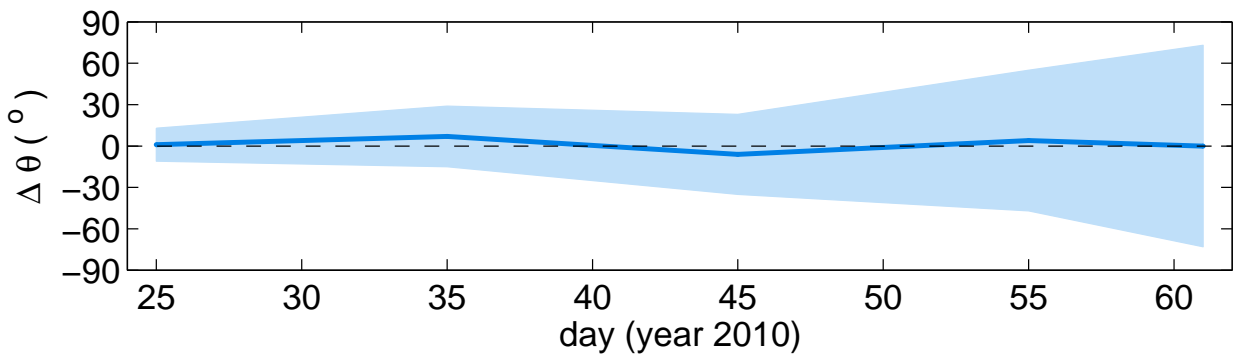

Figure 14: Time series of flow direction discrepancies averaged over the areas of the attracting LCS of $G_{1}$-filtered cases (occupied by the tracer) for (a) $\lambda_{C}=20 \mathrm{~km}$, (b) $\lambda_{C}=40 \mathrm{~km}$, and (c) $\lambda_{C}=80 \mathrm{~km}$, respectively. The shading areas are delimited by the standard deviations. 


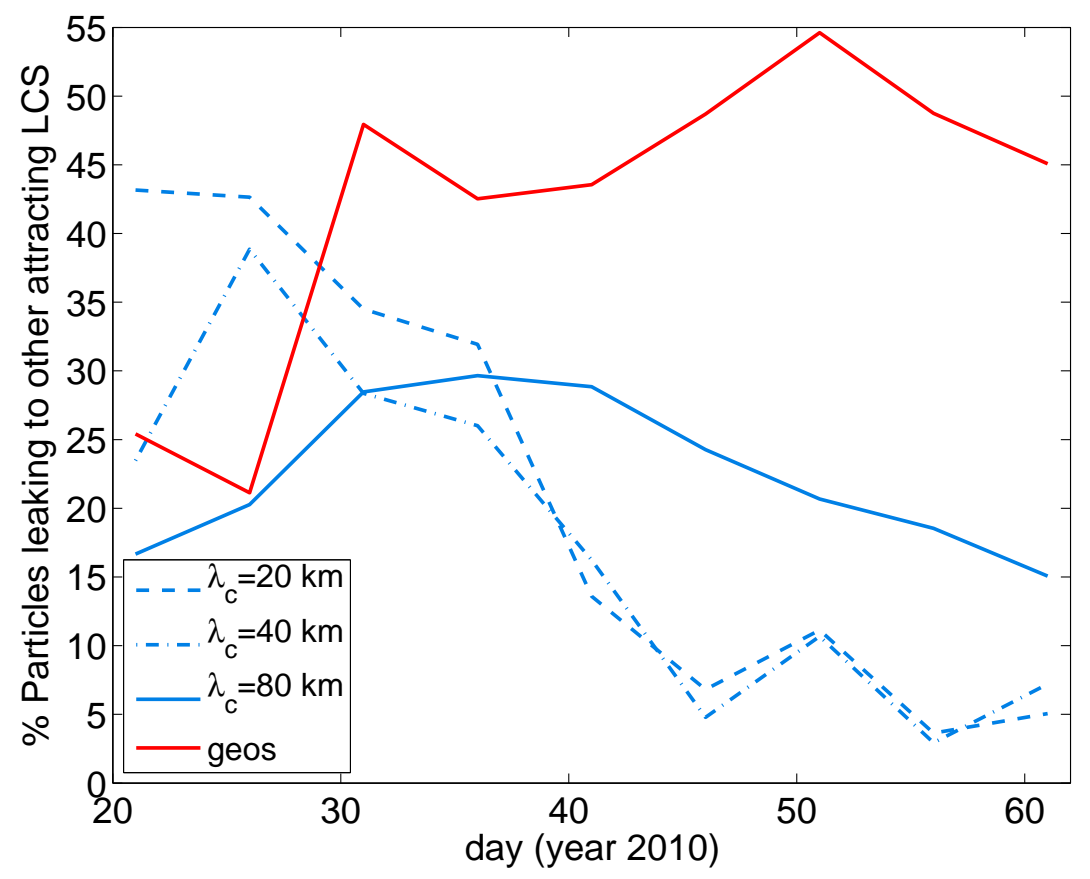

Figure 15: Time series of percentages of the total number of particles having leaked to other attracting LCS unoccupied by the tracer for all filtered cases. 
(a)

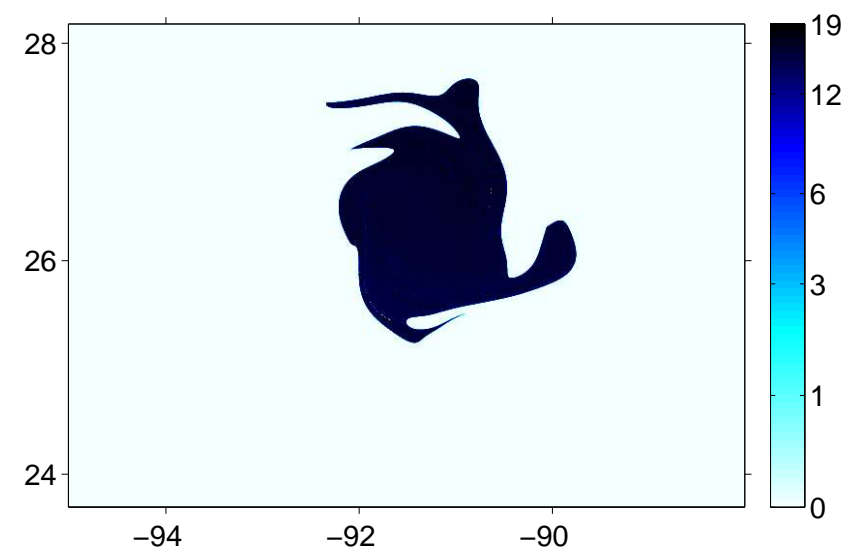

(b)

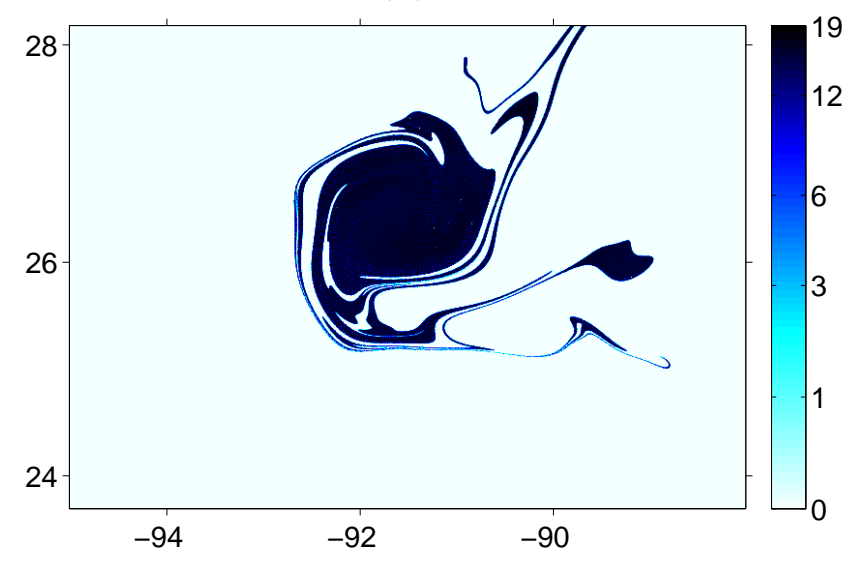

(c)

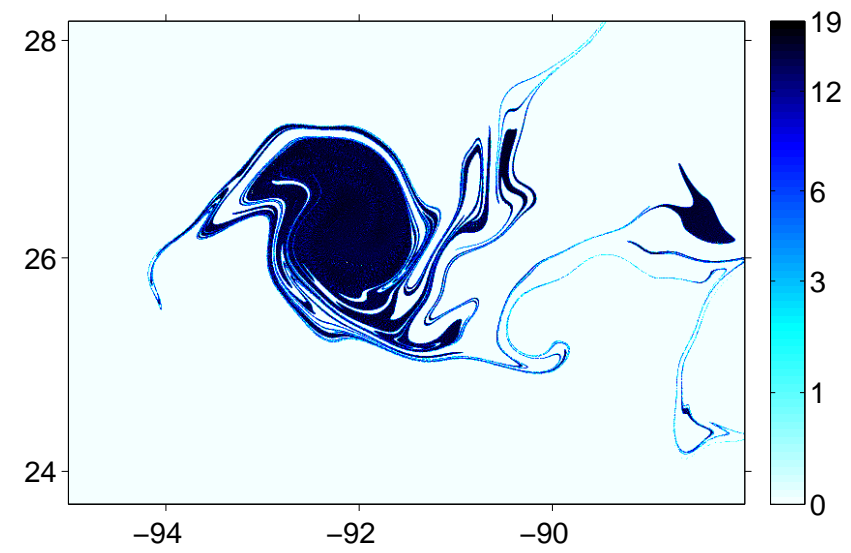

Figure 16: Particle concentrations on a log-scale 11 (top), 31 (middle) and 51 (bottom) days after a square-launch centered on the Star Eddy, advected by the geostrophic velocities from the altimetry-proxy. 
(a)

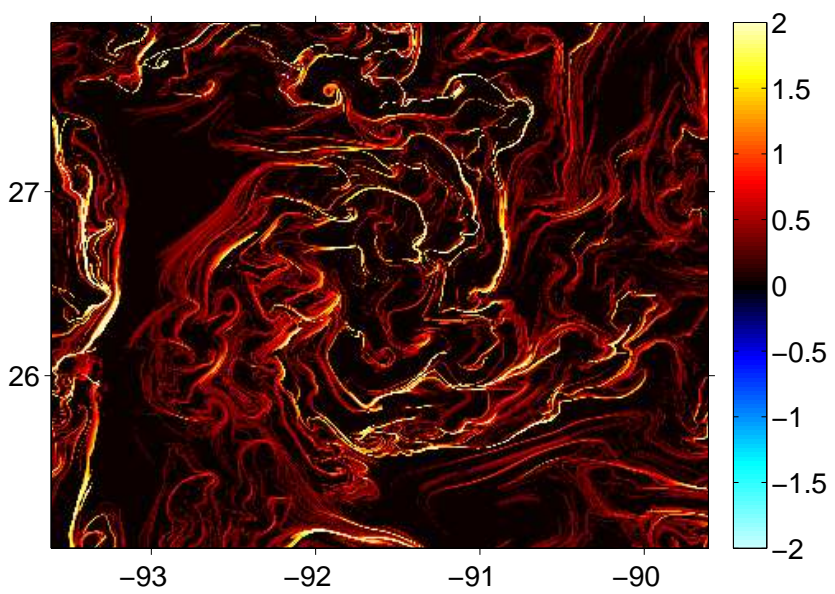

(b)

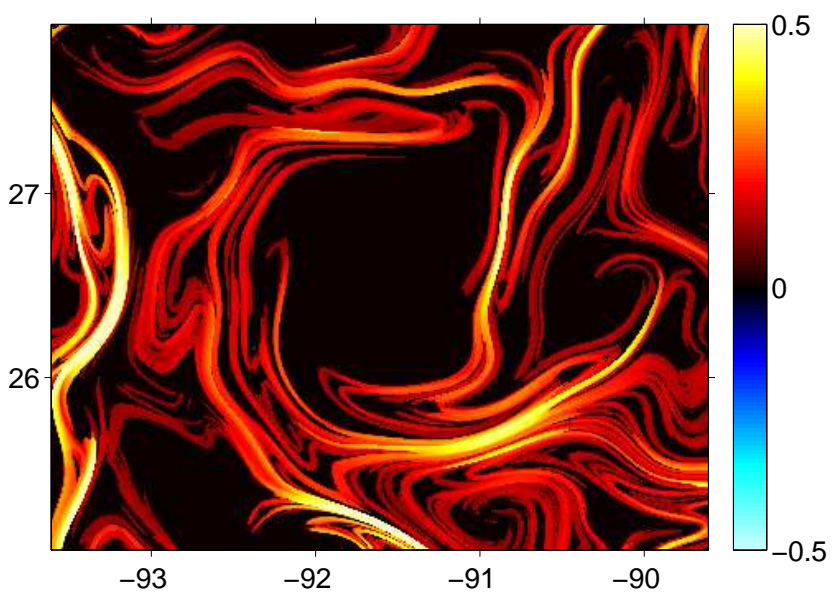

(c)

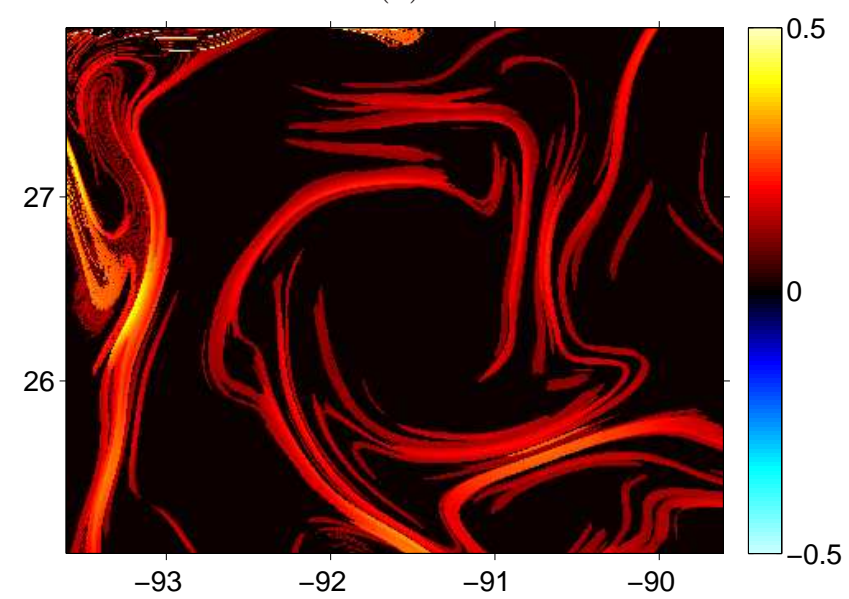

Figure 17: Attracting LCS (backward in time FSLE) at day=35 computed from the full velocity field (top), the Lanczos filtered fields with $\lambda_{C}=80 \mathrm{~km}$ (middle), and the geostrophic velocities of the altimetry proxy (bottom). 
year 2010, day $=15$
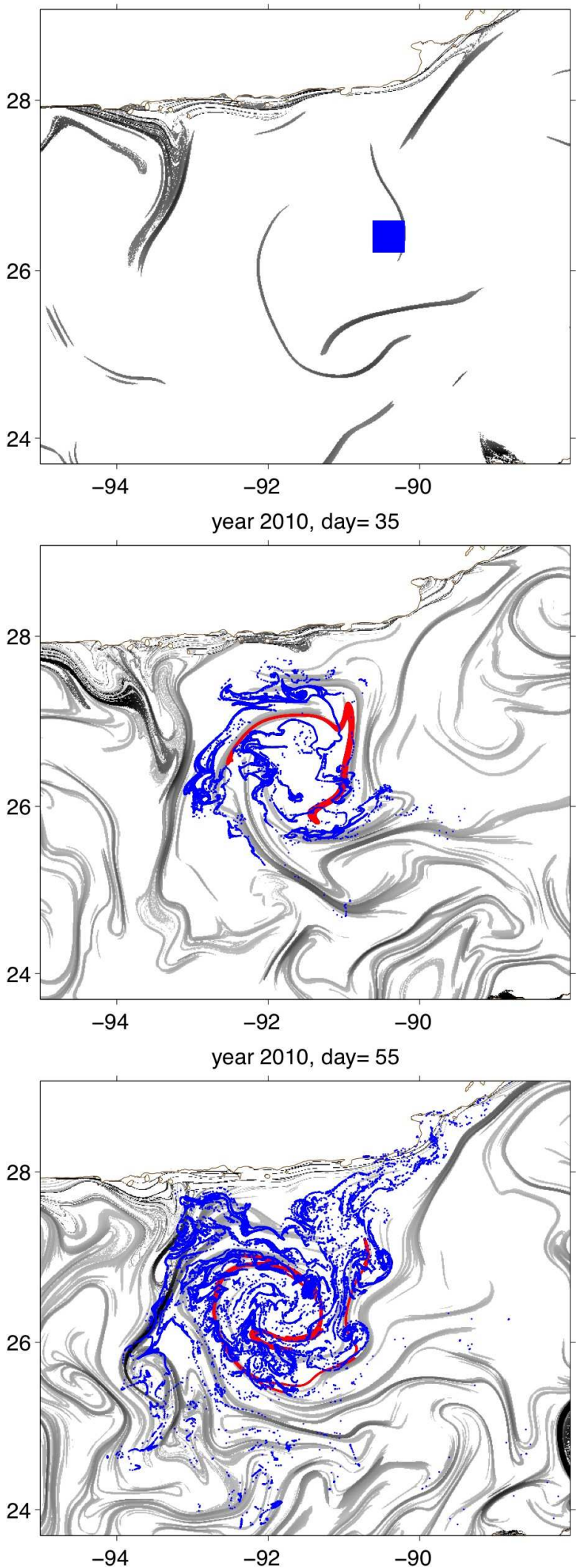

year 2010, day $=25$

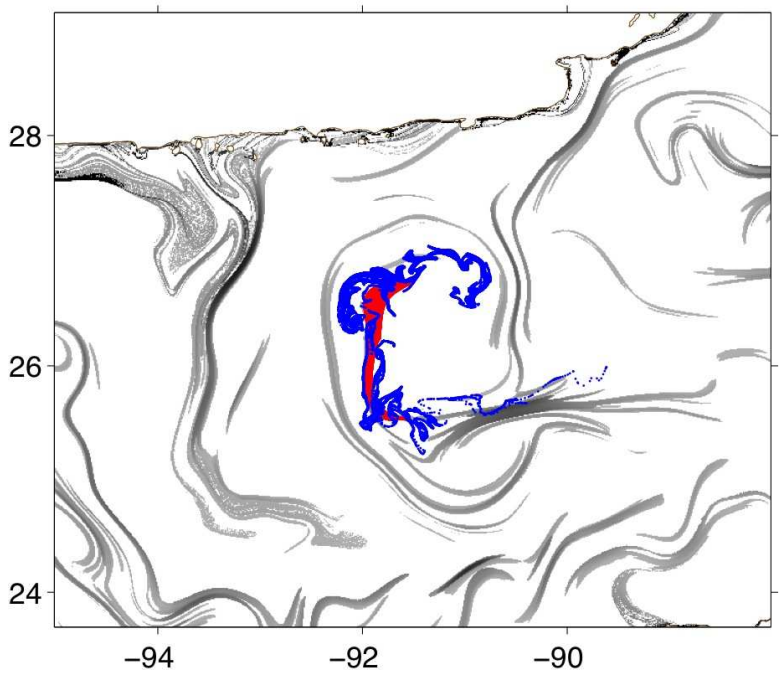

year 2010, day $=45$

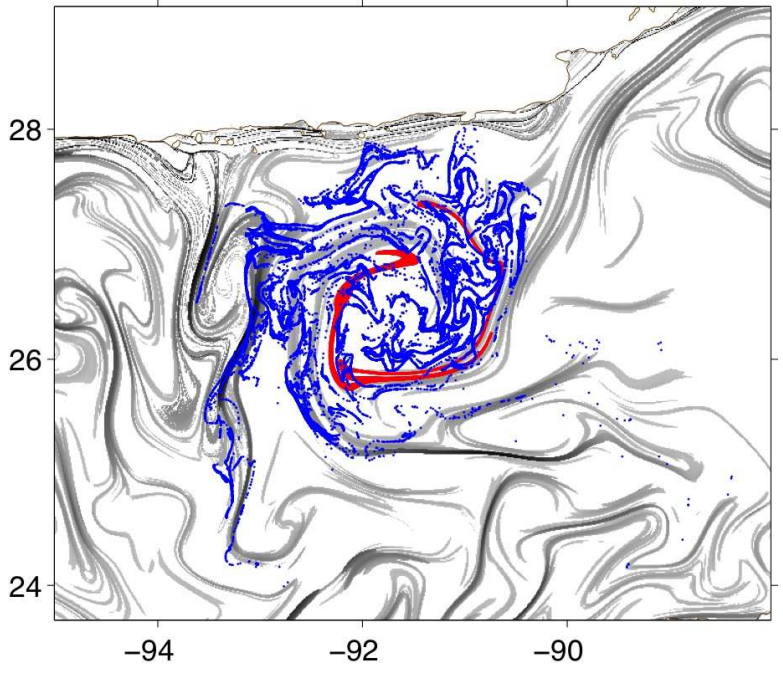

year 2010, day $=61$

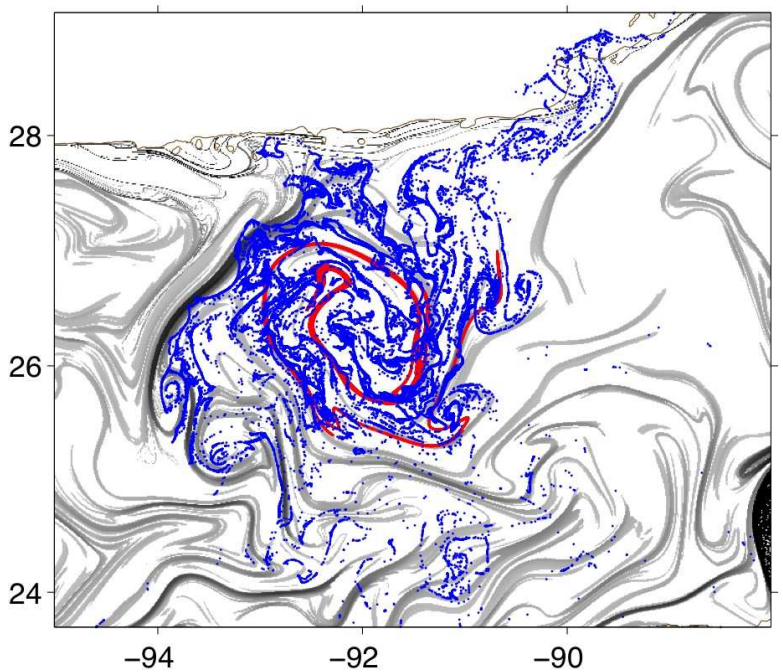

Figure 18: Same as Fig. 11 but the FSLE ridges and red particles are derived from field filtered by $G_{2}$, while the blue tracer shows the tracer advection by the full field. 

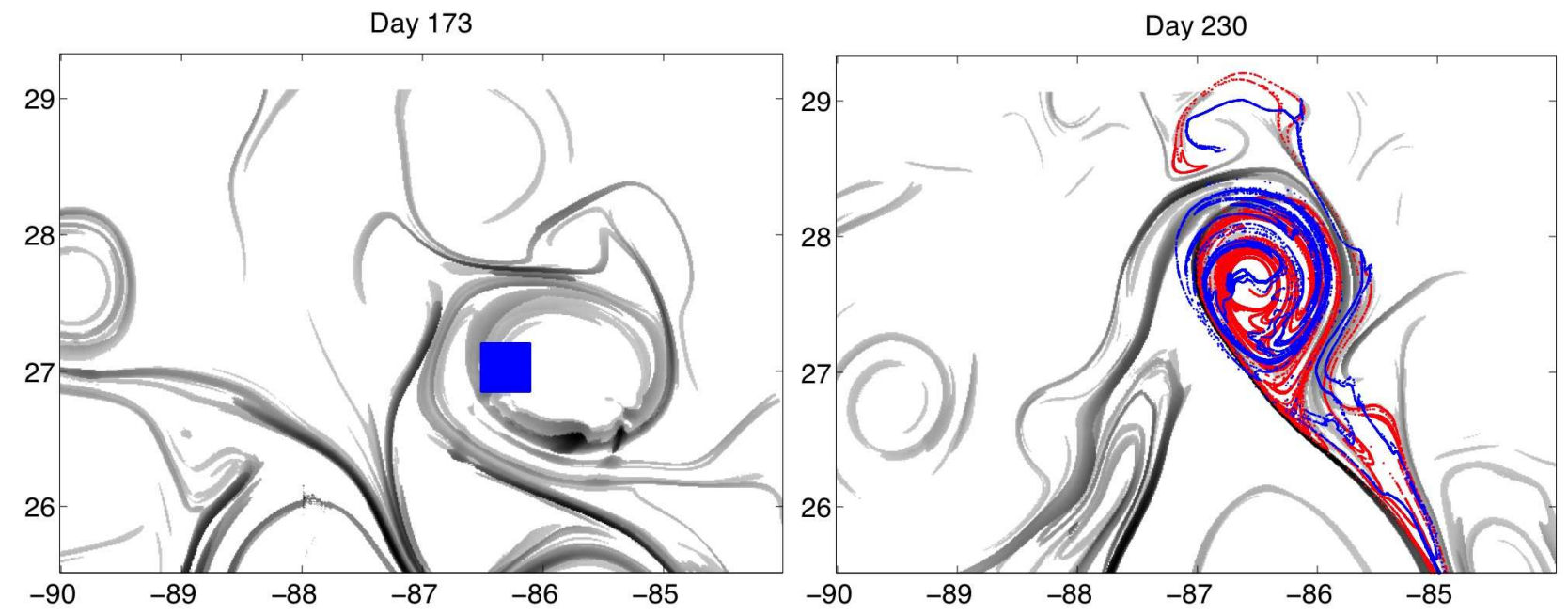

Figure 19: (Left Panel) Tracer release location on year day = 173 (June 22) in an eddy in the HYCOM GoM simulation. (Right Panel) Tracer distributions from the full (blue) and filtered (with $\lambda_{C}=80 \mathrm{~km}$ ) velocity fields (red) after 57 days (August 18). 